\title{
Simulation of spontaneous and variable global dust storms with the GFDL Mars GCM
}

\author{
Shabari Basu, ${ }^{1}$ John Wilson, ${ }^{2}$ Mark Richardson, ${ }^{3}$ and Andrew Ingersoll ${ }^{3}$ \\ Received 6 December 2005; revised 20 March 2006; accepted 2 June 2006; published 2 September 2006.
}

[1] We report on the successful simulation of global dust storms in a general circulation model. The simulated storms develop spontaneously in multiyear simulations and exhibit significant interannual variability. The simulated storms produce dramatic increases in atmospheric dustiness, global-mean air temperatures, and atmospheric circulation intensity, in accord with observations. As with observed global storms, spontaneous initiation of storms in the model occurs in southern spring and summer, and there is significant interannual variability in storm development: years with no storms are interspersed with years with storms of various sizes and specific seasonal date of initiation. Our results support the idea that variable and spontaneous global dust storm behavior can emerge from a periodically forced system (the only forcing being the diurnal and seasonal cycles) when the dust injection mechanism involves an activation threshold. In our simulations, surface wind stresses associated with resolved, large-scale $(>300 \mathrm{~km})$ wind systems initiate the storms. These winds are generally associated with the seasonally migrating $\mathrm{CO}_{2}$ cap boundary and sloping topography of the Hellas basin, thermal tides, and traveling waves. A very limited number of large storms begin with lifting along the frontal zones associated with traveling waves in the northern hemisphere.

Explosive growth to global scales results from the intensification of the Hadley circulation and the activation of secondary dust-lifting centers.

Citation: Basu, S., J. Wilson, M. Richardson, and A. Ingersoll (2006), Simulation of spontaneous and variable global dust storms with the GFDL Mars GCM, J. Geophys. Res., 111, E09004, doi:10.1029/2005JE002660.

\section{Introduction}

[2] Planetary-scale storms are major atmospheric events observed for months in the Martian atmosphere. At their peak, these storms are so extensive that they were observed on occasion through terrestrial telescopes decades before they were documented in detail from orbiting spacecraft [Leovy et al., 1972; Briggs et al., 1979; Kahn et al., 1992; Zurek and Martin, 1993; Martin and Richardson, 1993; Fenton et al., 1997; Smith et al., 2002; Liu et al., 2003]. During such a great dust storm (GDS) [Gierasch, 1974], the atmospheric haze becomes sufficiently thick that surface features become almost completely obscured, as was the case for weeks after the arrival of the Mariner 9 spacecraft at Mars in 1971, and most recently during the 2001 GDS. The presence of large amounts of dust in the atmosphere during a GDS modifies atmospheric temperatures and the global circulation. The dust is a mineral aerosol [Kahn et al., 1992]

\footnotetext{
${ }^{1}$ Department of Atmospheric Science, Texas A\&M University, College Station, Texas, USA.

${ }^{2}$ Geophysical Fluid Dynamics Laboratory, National Oceanic and Atmospheric Administration, Princeton, New Jersey, USA.

${ }^{3}$ Division of Geological and Planetary Sciences, California Institute of Technology, Pasadena, California, USA.
}

Copyright 2006 by the American Geophysical Union. 0148-0227/06/2005JE002660 that interacts with visible and infrared radiation, modifying atmospheric heating rates. By influencing heating rates, and hence atmospheric temperatures and circulation, GDSs may modify the cycles of water and $\mathrm{CO}_{2}$ on Mars. Observations over the course of the past century or so suggest that GDSs occur only during southern spring and summer. Globalmean air temperatures show highly repeatable behavior in northern spring and summer, with the clear indication of GDS activity in southern spring and summer [Liu et al., 2003; Smith, 2004]. This timing is consistent with greater forcing of the Martian atmosphere during the period around perihelion (at $L_{S}=251^{\circ}$ ). The observations also show that global storms do not occur every Martian year. On the basis of spacecraft and telescopic observations, Zurek and Martin [1993] estimated that a GDS occurs once every two to three Martian years. Even in years with global events, the size and exact season of their occurrence vary significantly: The 2001 storm went global at $L_{s}=186^{\circ}$, while the $1977 \mathrm{~b}$ storm began just after southern summer solstice. Understanding of the Martian climate requires an understanding of the dynamics of these variable phenomena.

[3] There is a spectrum of dust storm activity on Mars, with global-scale storms being the largest and most spectacular. Regional-scale storms typically occur in one or two seasons every year. In the northern hemisphere, these storms appear to be associated with traveling waves during the fall and winter seasons $\left(L_{S}=210^{\circ}-235^{\circ}\right.$ and $\left.L_{S}=310^{\circ}-345^{\circ}\right)$ 
[Cantor et al., 2001; Wang et al., 2005]. Cap edge storms are ubiquitous and Hellas is typically a rather active location.

[4] Triggering and storm shut-off are key puzzles behind GDSs: What makes a tiny fraction of the many small dust storms that occur each year grow into globalscale events, why do they only occur in some years, and why are the sizes and timing of occurrence different from year to year? Hypotheses for triggering rely on radiativedynamical feedback in which the lifted dust plays a key role. Leovy et al. [1973] proposed that the superposition of the Hadley cell circulation, the planetary-scale topographic winds, and the thermal tide would produce wind speeds high enough to raise dust from the surface. Pankine and Ingersoll [2002] demonstrated in their low order model that stochastic resonance due to prescribed weather noise was responsible for the triggering of these global storms.

[5] The variability of GDS triggering can arise in two ways: internal variability in the atmospheric dynamics (involving the circulation elements) and the development of a memory in the boundary conditions, say, albedo or surface dust reservoir. The mechanisms delineated above likely would not repeat perfectly each year, due to a variety of circumstances, depending on the dynamics of the particular system in question. This bias is borne of our experience of weather: only in special circumstances is weather highly predictable. This internal variability in the Martian atmosphere is only significant for GDS development if the threshold for storm growth falls somewhere in the range of naturally occurring variability [Newman et al., 2002a, $2002 \mathrm{~b}$ ]. In this case, internal perturbations (such as a strong coherence of dynamical systems, as suggested by Leovy et al. [1973] and most recently by Wang et al. [2003]) could push the atmospheric system into a state that generates a GDS. Pankine and Ingersoll [2002] illustrated this idea with a low-order numerical model forced with prescribed noise. However, it is possible that GDS variability is not limited solely or at all by low-probability weather events. Availability of dust on the surface may be a key factor, or it may be that spatial redistribution of dust on the surface (or ice in the seasonal polar caps) modifies the forcing of the circulation in such a way as to allow GDSs in some configurations, but not others. In both cases, these changes in the surface are sensitive to the history of dust activity in previous years [Haberle, 1986]. The major question we wish to address here is whether internal variability in a general circulation model (GCM) can generate "weather noise" of the right character to allow the first means of variability to operate. The issue of the role of surface memory for interannual and intraseasonal variability of global storms is the subject of a subsequent paper. We are looking to "tune" our model so that weather noise is sufficient to trigger substantial lifting with interannual variability. Hence it is important to characterize the weather noise, which is almost exclusively from traveling waves in fixed dust simulations (R. J. Wilson et al., manuscript in preparation, 2006). If dust is allowed to vary interactively, then one imagines that tides and convective dust lifting, which depends on static stability, are also variable [Newman et al., 2002a, 2002b]. For the case of Hellas storms (which is the source region for all our major storms), both of these come into the picture.

[6] Work on the numerical modeling of global storms began with investigations of the degree to which dust can influence the Martian circulation. Two- and threedimensional modeling showed that the atmosphere responds quite dramatically and there exists ample opportunity for nonlinear feedback in the system once dust is injected [Haberle et al., 1982; Murphy et al., 1993, 1995; Wilson, 1997]. These modeling efforts also showed that once a significant amount of dust is injected into the atmosphere, global-mean air temperatures and the signatures of the thermal tide in pressure and temperature observations could be explained reasonably well throughout the storm decay phase [Murphy et al., 1993, 1995; Wilson and Hamilton, 1996; Wilson, 1997; Wilson and Richardson, 2000]. Results of experiments with interactive dust lifting, where dust injection is controlled by model-resolved winds and near surface static stability, were published by Newman et al. [2002a, 2002b]. The Newman et al. [2002a, 2002b] work demonstrated that a range of dust storm activity could emerge from the natural steady forcing of a GCM by diurnal and seasonal cycles. However, the questions outlined above remain open following the Newman et al. [2002a, 2002b] work; their model was not able to obtain global dust storms in a realistic way (while "global" dust events were simulated, they corresponded to unrealistic dust injection scenarios discussed in the next section), and the year-to-year variability in dust activity was very muted compared with observations. Simulating the emergence of GDSs from a realistic background state is critical for correctly representing the Martian dust cycle.

[7] The importance of the background cycle was not addressed by Newman et al. [2002a, 2002b]. The simulation that they suggest might be able to best represent all the aspects of the Martian dust cycle has abnormally high opacities during northern spring and summer season and little interannual variability compared to observations. This is due to threshold dependent dust devil lifting (DTH) that is a major contributor in their parameterization along with stress lifting. Even though DTH helps in realistic shut-off of storms due to a negative feedback effect, it also gives rise to unrealistic opacities in some seasons. With the combined threshold sensitive stress lifting and the dust devil only lifting scheme, they obtained intense lifting at $\sim L_{s}=190^{\circ}$ in the Hellas region and these are some attributes that have been observed in 2001 global dust storm. However, high dust opacity scenarios could not be explored as their model reached its stability limits due to explosive opacity increases. Also the cross-equatorial flushing type of storms [Wang et al., 2005], were not simulated accurately in terms of frequency and season of occurrence.

[8] For any realistic simulations of the Martian dust cycle it is important that the basic constraints in terms of temperatures and opacities are satisfied to first order for each season. In our study we find it is relatively easy to independently simulate the correct weather conditions (in agreement with observations) for a particular season or location. What is difficult is a good global representation of the Martian dust cycle, which can be achieved only when all the interactions between various lifting schemes, the 
boundary layer phenomena and the radiative dynamical feedback is formulated accurately.

[9] Basu et al. [2004] examined the Martian seasonal dust cycle with a GCM that treats dust as a radiatively and dynamically interactive trace species. This work takes off from Newman et al. [2002a, 2002b] studies. Dust injection is parameterized as being due to convective processes (such as dust devils) and model-resolved wind stresses. The seasonally-varying global air temperature derived from multiyear Viking and Mars Global Surveyor air temperature data sets is used to quantitatively assess the quality of simulations. It is found that northern spring and summer temperatures, which are observed to repeat very closely each year, can be reproduced by the model if the background dust haze was supplied by either convective lifting or by stress lifting with a very low threshold and a low injection rate [Basu et al., 2004]. In order for either of these cases to yield spontaneous and variable dust storms, dust injection due to high threshold, high rate stress lifting must be added. The convective scheme is found unable to generate a dust storm, from which it is concluded that dust devils do not initiate dust storms, in agreement with the conclusion reached by Cantor et al. [2002] based on MOC imagery. In order to supply the background haze, widespread and ongoing lifting is required by the model. Imaging data provide a viable candidate mechanism for this lifting if it is convective, in the form of dust devils. However, local storms and other observed, nonconvective lifting systems appear insufficiently frequent and widespread to satisfy the role demanded by the model. On the basis of the model results and inferences from thermal and imaging data, it is suggested that the seasonal cycle of background dust haze on Mars is maintained by the action of convective processes, and specifically dust devils.

[10] In this paper, we describe the simulation of global dust storms with the Geophysical Fluid Dynamics Laboratory (GFDL) Mars GCM using the dust-lifting parameterizations described by Basu et al. [2004]. By combining the convective scheme and high-threshold stress lifting, we can obtain a "best fit" multiyear simulation, which includes simulation of both a realistic thermal state in northern spring and summer and, for the first time, the spontaneous generation of interannually variable global dust storms. Our parameterization has 3 adjustable tuning parameters: dust devil lifting (DDL) rate $\left(\mathrm{R}_{\mathrm{DDL}}\right)$, stress lifting $(\mathrm{SL})$ rate $\left(\mathrm{R}_{\mathrm{SL}}\right)$ and stress threshold $\left(\tau_{\mathrm{SL}}\right)$ (we fix $\mathrm{R}_{\mathrm{DDL}}$ [Basu et al., 2004] and adjust the other two). We initially provide a sweep of dust injection parameters to map out the range of model behavior. Such a figure is presented by Basu et al. [2004, Figure 10]. This plot explores the range of phase space (stress lifting rate $\left(\mathrm{R}_{\mathrm{SL}}\right)$ versus stress threshold $\left(\tau_{\mathrm{SL}}\right)$ ) for which we carried out the simulations and discussed in section 3. It is found that realistic simulations of dust cycle with the background dust haze and spontaneous, interannually variable storms occur only for a narrow range of these parameters. Such fine-tuning can be attributed to the fact that similar climate is simulated every year and hence to amplify the little transience that exists in the simplistic model one has to really narrow down the choice of stress lifting parameters.

[11] We proceed to examine some of the multiyear simulations that generate spontaneous and variable global storms. The triggering and growth mechanisms are examined in some detail: global storms are primarily found to initiate in the southern midlatitudes near the Hellas basin in southern spring. A very limited number of large storms are found to initiate in the northern high latitudes in association with frontal cyclones, via the mechanism proposed by Wang et al. [2003]. In all cases, growth to global scales is found to require the entrainment of dust in the Hadley circulation and the subsequent activation of secondary dust-lifting centers.

\section{Model Description}

[12] The simulations discussed in this paper are undertaken with the GFDL Mars GCM, using the model in the form described by Basu et al. [2004]. Briefly, the model is a Mars-adapted version of the GFDL SKYHI GCM [Wilson and Hamilton, 1996]. For these simulations, grid point spacing is $5^{\circ}$ in latitude by $6^{\circ}$ in longitude, with 20 vertical levels between the surface and $\sim 85 \mathrm{~km}$. Radiative heating by dust and $\mathrm{CO}_{2}$ in the visible and thermal infrared is treated. The surface and boundary layers are treated with a Monin-Obukhov (MO) surface drag scheme coupled to a diffusive boundary layer with a Richardson numberdependent diffusion coefficient. Dust evolution is controlled by advection by large-scale winds, by subgrid diffusion, and by dust sedimentation. Currently we employ two dust particle sizes, $0.625 \mu \mathrm{m}$ and $2.5 \mu \mathrm{m}$, to represent a dust particle distribution. For simplicity, we assume that each particle size is characterized by the same optical properties. For these simulations, we use the values suggested by Clancy et al. [1995]; single scattering albedo, $\omega=$ 0.92 , and an asymmetry factor, $g$, of 0.55 . We can adjust the relative contributions of each particle size to total opacity. This is effectively equivalent to specifying different injection rates for each particle size. The sedimentation rates of these two are proportional to their radii, hence the bigger particles settle faster and the smaller particles have a greater residence time in the atmosphere. Water ice clouds are generally insignificant during the dust storm season [Smith et al., 2002]; hence we do not consider their possible scavenging and radiative impact.

[13] Dust is injected into the atmosphere using two schemes. The first represents small-scale, convective lifting, which observations suggest is primarily in the form of dust devil activity. This scheme relates the injection rate to the sensible heat flux and boundary layer depth (following Renno et al. [1998, 2000] and Newman et al. [2002a]), using a multiplicative, tunable injection rate parameter [Basu et al., 2004]. This rate parameter is labeled as $\mathrm{R}_{\mathrm{DDL}}$. The second scheme relates dust injection to the surface stress generated by model-resolved winds. The functional form sets the injection equal to the cube of the drag velocity scaled by a multiplicative, tunable rate parameter (labeled $\mathrm{R}_{\mathrm{SL}}$ ). There is no wind stress injection when the stress is below a threshold value. We regard this threshold value as a free parameter $\left(\tau_{\mathrm{SL}}\right)$. As such, there are three free parameters which provide the range of available exploration space: $R_{D D L}, R_{S L}$, and $\tau_{S L}$. The dust injection schemes, their behavior, and the ability of the GCM to simulate the observed annual cycle of midlevel air temperatures and dust column opacity are described in much greater depth by 


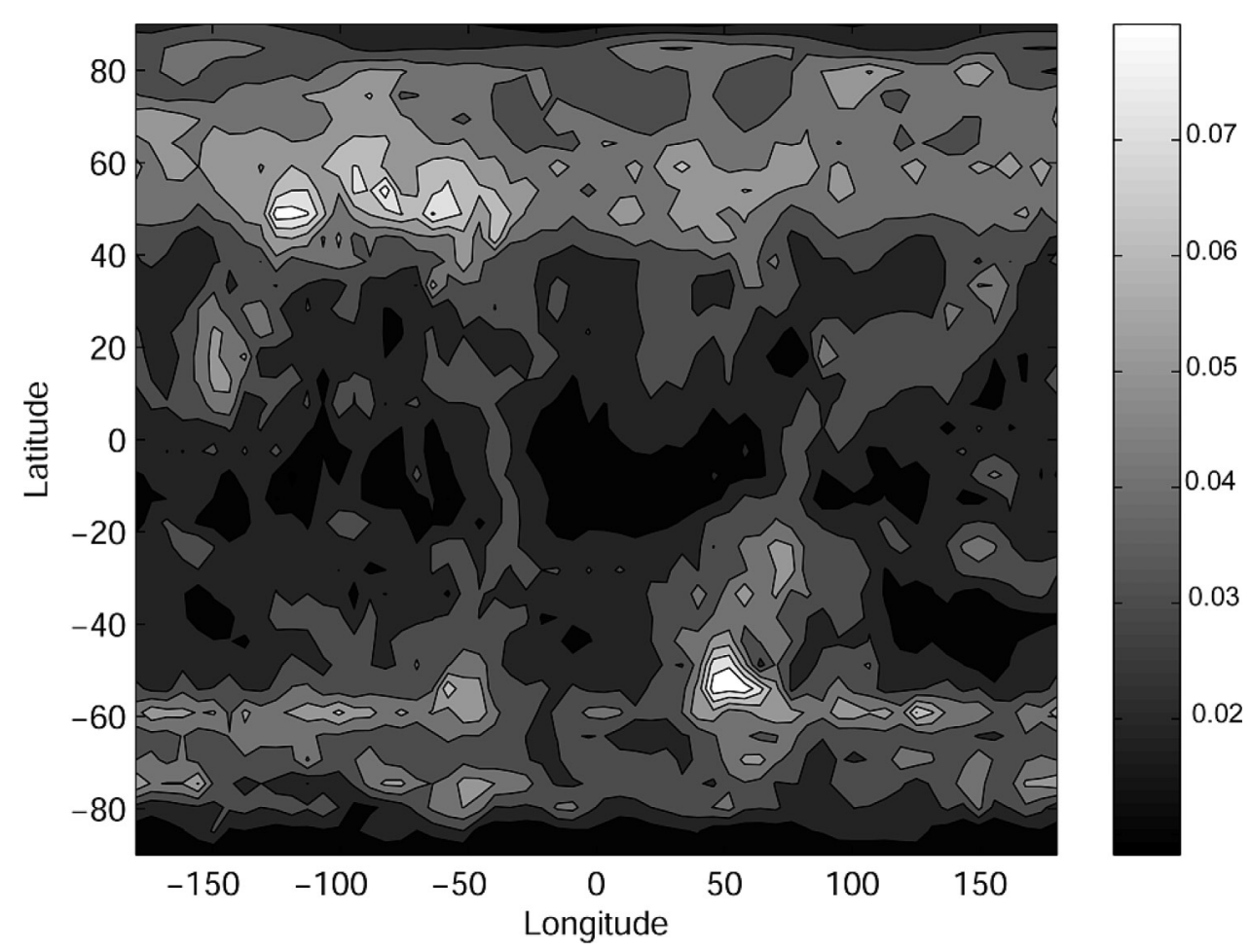

Figure 1. Geographic distribution of maximum stresses $(\mathrm{Pa})$ encountered at each grid point in a given annual cycle. The stresses are averaged over 10 years. No feedback other than convective lifting is active here. The high wind stresses are not uniformly distributed but are concentrated in specific regions, largely due to topography and the position of the wintertime baroclinic storm tracks. These are active dust-lifting regions. See Figure 6 for reference, where topography is contoured in black.

Basu et al. [2004]. Finally, dust is assumed infinitely available at the surface.

\section{Exploration of Model Behavior as Wind Stress Lifting Parameters Vary}

[14] The companion paper [Basu et al., 2004] describes the generation of a seasonal cycle of dust and air temperatures that are quite close to those observed. It should be emphasized that the envelope of globally averaged temperature smoothes out the two stormy periods $\left(L_{S}=210^{\circ}-235^{\circ}\right.$ and $\left.L_{s}=310^{\circ}-350^{\circ}\right)$ that are apparent in TES temperatures. These periods have regional-scale dust storms associated with traveling wave activity in the northern hemisphere that can result in flushing storm behavior. An important result from that paper, of relevance to this study, is the finding that convective processes must provide the majority of the dust injection during the seasons when regional storms are rare. We found that for wind stress lifting to play a major role, the stress threshold has to be lowered to a point wherein local and regional storms are essentially continuously ongoing over a large fraction of the planetary surface. This is inconsistent with observations [Briggs et al., 1979; Cantor et al., 2001]. As the parameters are changed to generate fewer and bigger dust storms, more in keeping with the observations, the model produces an air temperature cycle that is increasingly discordant with observations [Basu et al., 2004]. This is a useful result for our purposes as it allows us to separate convective and wind stress lifting. Even though Newman et al. [2002a, 2002b] employed a variable threshold scheme based on semiempirical formulae, we chose to apply a uniform stress threshold scheme for the entire planet. This makes the parameterization simpler and helps us understand the phenomena involved in the evolution and interannual variability of these storms rather than getting distracted with the details of specific regional storms.

[15] The convective lifting can be tuned to fit the air temperature cycle (by choosing a spatially and temporally fixed value of $R_{D D L}$ for use in all of our subsequent simulations), leaving only a range of values for $R_{S L}$ and $\tau_{\mathrm{SL}}$ to be explored for dust storm behavior. If we allow stress lifting to generate the background dust, and ignore the overgeneration of local and regional storm activity, we find that there is no development of global storms and essentially no interannual variability.

[16] A spatial map of the maximum wind stress experienced at different places on the planet in a no dust storm year is also helpful in determining the critical range of parameters without actually running simulations for each set of tunable parameters. Figure 1 shows such a map for 10 consecutive simulated years with convective lifting only. This experiment yields a very good simulation of the non dust storm Martian climate [Basu et al., 2004]. This indicates the regions where we expect dust lifting to be most likely and is reasonably the basis for tunings of the model, especially the stress threshold. It is clear from Figure 1 that high wind stress is not uniformly distributed, but concentrated in specific regions, largely due to topography and the position of the wintertime baroclinic storm 


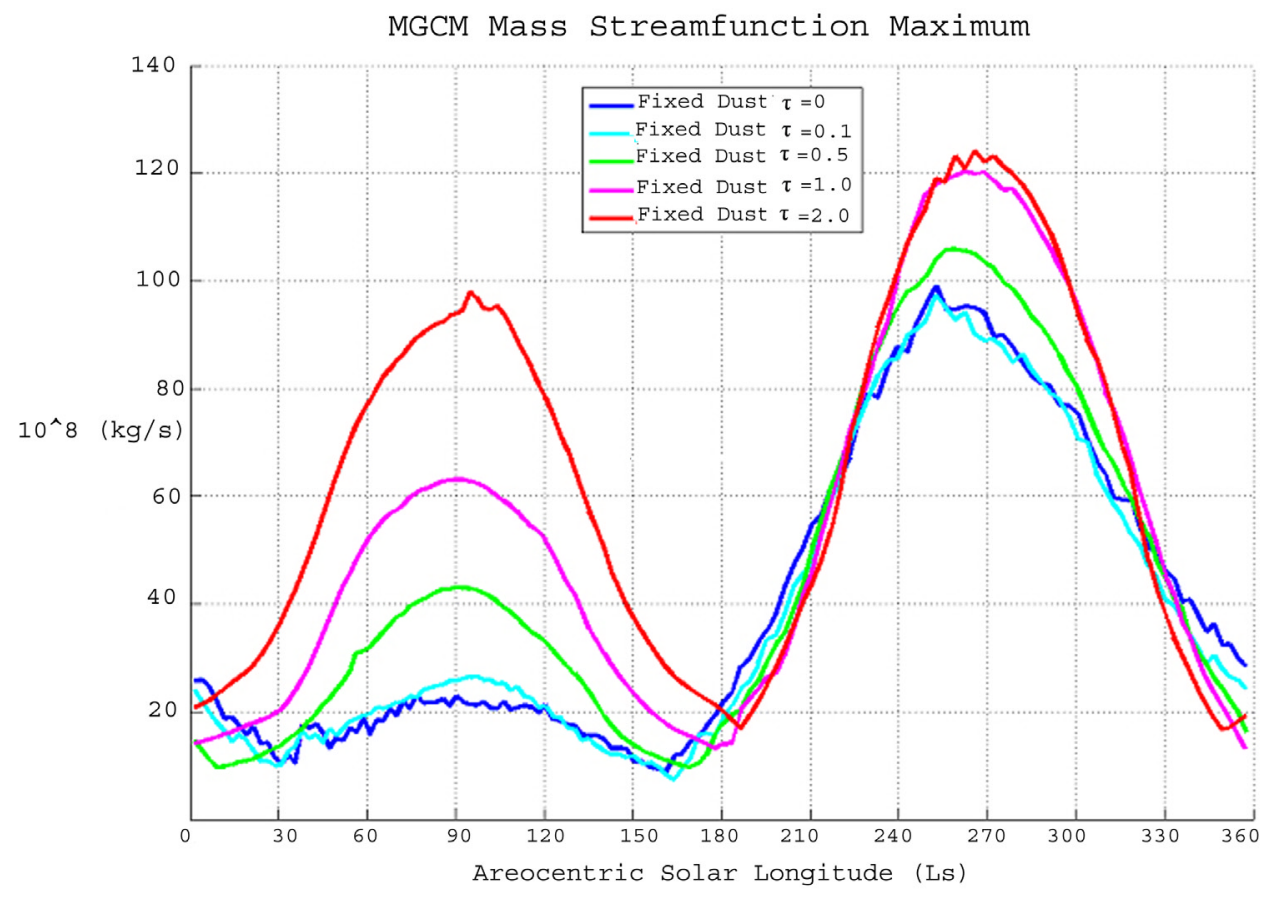

Figure 2. The seasonal variation of maximum absolute value of the mass transport stream function for a series of different dust optical depths. The maximum value is around roughly $14 \mathrm{~km}$ above the surface in the tropics.

tracks. The southwest corner of the Hellas region is particularly prominent along with the Alba Patera region. As we will discuss in the following sections, these regions are sources of two major types of storms that we simulate. The Hellas basin is dominated by tides in the southern summer season. The stresses during this time are dominated by diurnal variations and there is not much other variability. The subtropical jet (along a zonal collar at $30^{\circ} \mathrm{S}$ ) helps in obtaining higher stresses in the basin. This jet is connected to the Hadley cell circulation, and has a strong seasonality [Haberle et al., 1993; Joshi et al., 1995]. This is also reinforced by upslope winds in the basin. The band of high stresses in the southern hemisphere follows the $\mathrm{CO}_{2}$ ice cap. These high stress regions are the dominant source regions for storms as there is a tendency for dust to be exported from these locations when the stress threshold is exceeded (see section 6). The Syria and Solis Planum regions are characterized by rather weak surface stresses, and hence they do not contribute to the storms in our simulations. Observationally, the high stress regions are correlated with the low-albedo regions [Ruff and Christensen, 2002].

[17] There is a maximum variability in the stresses in the Hellas region during equinox, $L_{s} \sim 180^{\circ}$. Much of this is due to traveling waves. However, these stresses do not give rise to global-scale storms since the Hadley cell circulation is quite weak in this season. The Hadley cell circulation is a critical component of the modeling presented in this paper. This study is about the amplification of a small noise signal and the Hadley circulation is the prime amplification mechanism. Seasonal variations are much more pronounced on Mars. At the solstices, the Hadley circulation is dominated by single cross-equatorial cells (Figure 13), while at the equinoxes, two less intense cells emerge. Figure 2 shows the seasonal variation of the Hadley cell circulation for a few different fixed dust scenarios. This emphasizes the importance of season for the intensity of circulation. The Hadley cell is the strongest $\sim L_{s}=270^{\circ}$. If the dust storm gets entrained into the circulation by this time, positive feedback between the circulation and the lifted dust ensues and helps in the explosive growth of the storm. The strong seasonal cycle is most sensitive to the displacement of the subsolar latitude poleward from the equator, as described in theory [Lindzen and Hou, 1988]. Interestingly, there is greater sensitivity during northern spring and summer.

[18] The dust cycle and dust storm behavior in each of our simulations has been assessed by examination of the globally averaged, mid-level $(\sim 25 \mathrm{~km})$ air temperature $\left(T_{15}\right)$ cycle, and the evolving behavior of the spatial variation of dust (undertaken by examining "movies" of dust distribution maps). Example air temperature cycles are shown in Figures $3 a-3 c$. The comparison climatology is derived from seven Martian years of spacecraft thermal infrared data [Liu et al., 2003; Basu et al., 2004]. Figure 3a shows output from a simulation with a relatively low value of the threshold stress for lifting $\left(\tau_{\mathrm{SL}}\right)$. In this particular case, the injection rate parameter is set high enough that global dust storms are generated in the model. However, the simulated storms are found to repeat almost exactly in each year. It should also be noted that in northern spring, the shape of the simulated air temperature cycle deviates slightly from observations, with a shift of the temperature minimum to near $L_{s}=90^{\circ}$. This is because the Hadley cell leads to a tropical temperature minimum at $L_{s}=90^{\circ}$. However, TES finds a minimum closer to $L_{s}=45^{\circ}$ because cloud heating becomes increasingly significant in the solstice season when the cloud belt is the thickest. Very high values of stress threshold and low 


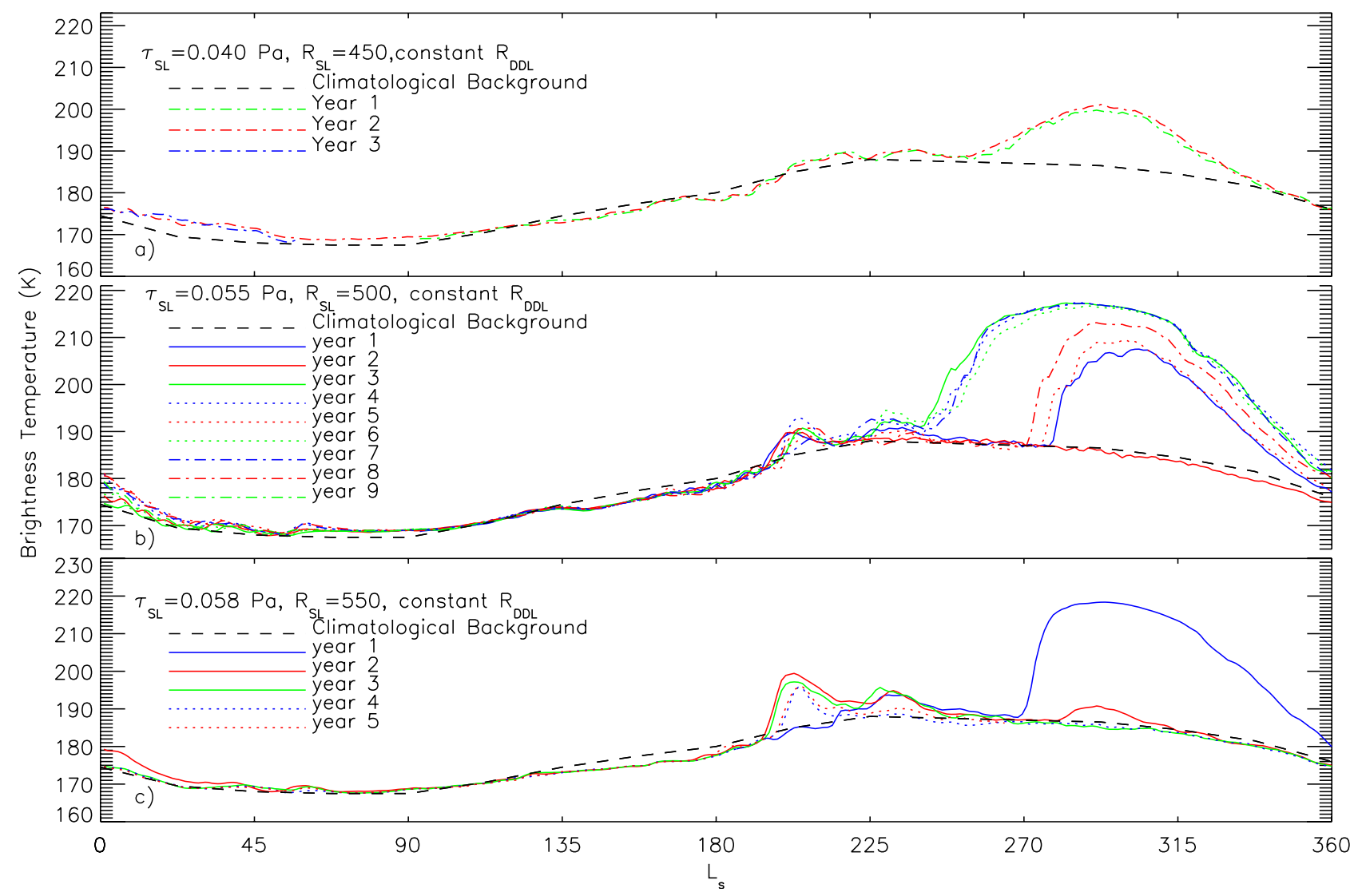

Figure 3. Multiyear globally averaged $T_{15}(\mathrm{~K})$ temperatures as a function of areocentric solar longitude: (a) low threshold case of $0.04 \mathrm{~Pa}$, (b) critical threshold case of $0.055 \mathrm{~Pa}$, and (c) threshold case of $0.058 \mathrm{~Pa}$.

values of injection rate leads to essentially no stress lifting, no dust storms, and essentially no interannual variability.

[19] Figure 3b shows an example of variable global dust storm generation in the model. In this case, the stress threshold and injection rate parameter are set such that realistic local and seasonal dust storms develop [Basu et al., 2004], but, in addition, global dust storms with peak temperatures close to those observed can develop in southern spring and summer. Critically, unlike the case in Figure 3a, the global dust storms do not repeat every year, are not the same size nor begin at exactly the same seasonal date each year. As the storms decay, air temperatures relax back to a realistic state, and the following year may or may not have a global storm. The details of these storms are discussed in the next section.

[20] Using the criteria laid out in the previous paragraph, the archive of simulations was examined to search for the development of global dust storms, variability of these storms, and the quality of air temperature predictions for northern spring and summer. Only the stress lifting injection rate $\left(R_{S L}\right)$ and the stress threshold $\left(\tau_{S L}\right)$ were varied between simulations, and all parameters were held constant during each individual simulation. The results are summarized by Basu et al. [2004] and also shown in Figure 4. Different shapes are used to represent different model behaviors. This figure shows several distinct and coherent domains of behavior. For low injection rate $\left(\mathrm{R}_{\mathrm{SL}}\right)$, the stress lifting does not significantly impact the simulations: no global storms are generated, and insufficient dust is lifted to cause the modeled dust and air temperature cycle to deviate from that generated by the convective lifting scheme. This domain of no global dust storm behavior eventually terminates as the injection rate is increased to a high enough value. This "termination" injection rate has higher values for higher threshold stresses. This makes sense: when a smaller fraction of modeled winds can loft dust, the model requires higher lifting rates for a given stress value to loft the same mass of dust. At the other extreme in the phase space, if the injection rate is set too high, the atmosphere is incapable of moving this dust out of the lowest atmospheric level ( 200-300 $\mathrm{m}$ thick) as the removal rate is much lower compared to the injection rate. While these simulations can still be carried forward, and a subset generate northern summer air temperatures that are not too unrealistic, the lowest-level opacities become unrealistically high. In some of these simulations "global dust storms" can be generated, but not within a global and annual context that is in any sense realistic. The fine-tuning of these parameters is required to get realistic variable global dust storms. A thin wedge of domain space, constrained mainly by the lifting rate parameter, separates underprediction and overprediction of wind stress dust lifting.

[21] This thin wedge of domain space where global dust storms occur within the context of a somewhat realistic model state can itself be further subdivided. For values of threshold stress that are too low, global dust storms develop, 


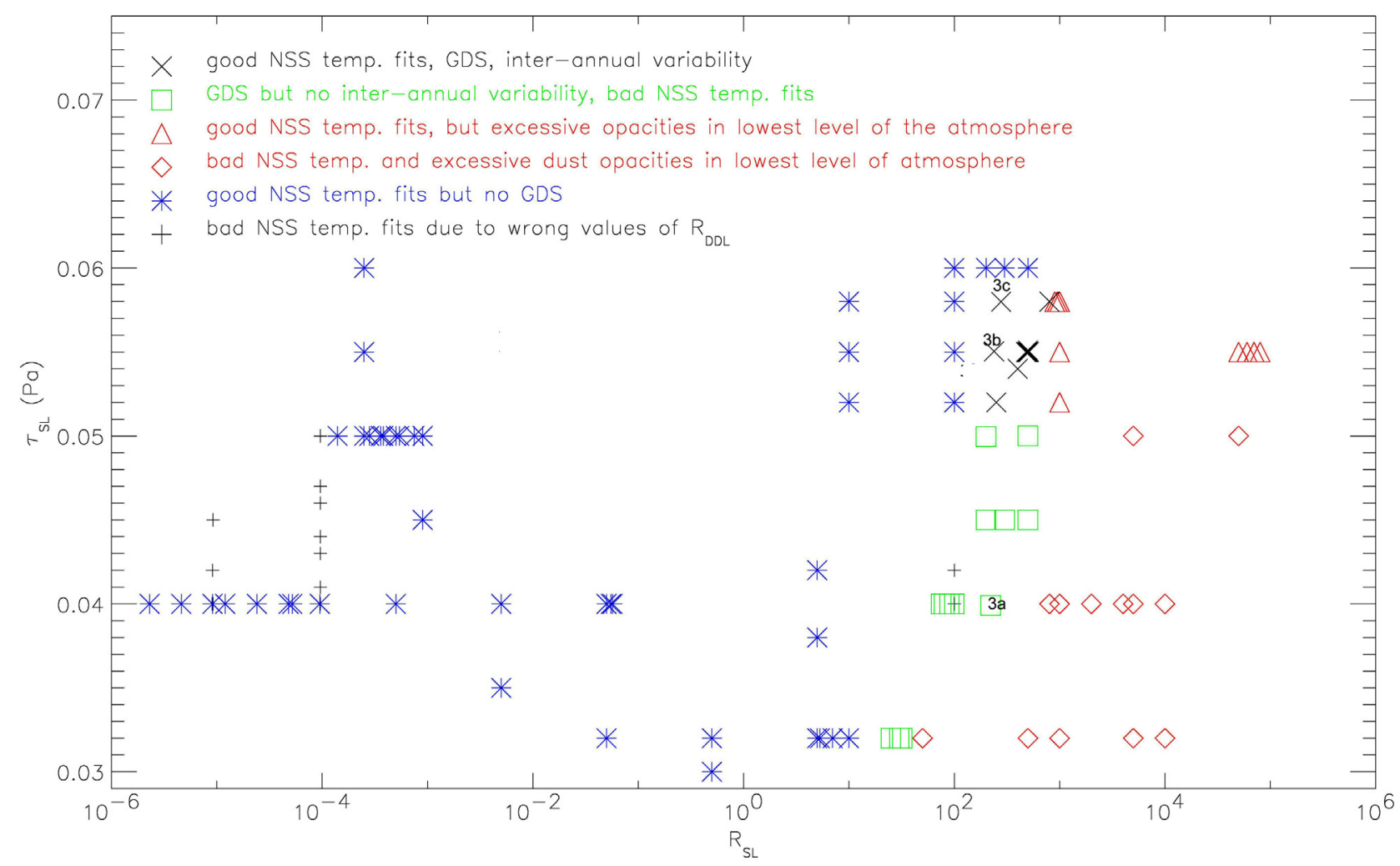

Figure 4. A summary of the wind stress scheme phase space examined for various dust storm behavior. In all cases, the best fit DDL injection parameters were used, and only a single set of wind stress parameters were used in a given simulation. In a specific area of phase space (indicated), spontaneous and variable global dust storms were simulated in southern summer, emerging from and returning to realistic non dust storm states in northern spring and summer (NSS). Significantly, these simulations exhibited years with and without global dust storms within the same multiannual simulation as shown in Figure 3. The points labeled 3a, 3b, and 3c correspond to the three simulations shown in Figures 3a, 3b, and 3c.

but without interannual variability. In many of these cases, as shown in Figure 3a, the northern spring air temperatures are not well simulated (these simulations are distinct from those mentioned above with excessive lifting rates in that these simulations do not generate events like global dust storms in northern summer, which are totally at odds with observations). Only in a limited area of the $R_{S L}-\tau_{S L}$ parameter-space do spontaneous and variable global dust storms develop. For $\mathrm{R}_{\mathrm{SL}}$ and $\tau_{\mathrm{SL}}$ values that are neither too large nor too small, the model is able to generate simulations resembling those shown in Figure 3b. Within this area, variations in the parameters, especially the stress threshold, cause variations in the nature of the simulated global storms. Figure $3 \mathrm{c}$ shows an example for a threshold of $\tau_{\mathrm{SL}}=$ $0.058 \mathrm{~Pa}$, to be contrasted with the simulation shown in Figure $3 \mathrm{~b}\left(\tau_{\mathrm{SL}}=0.055 \mathrm{~Pa}\right)$. In the Figure $3 \mathrm{c}$ case, there is some variability in the evolution of the storms, including some evidence for multiple, Hellas regional storms in a single year. When the stress threshold is raised, the lifting rate also goes up, as now the lifting regions are being limited and hence for significant dust lifting the rate has to be higher. In the southern hemisphere spring season, high stresses are attained in Hellas basin due to traveling waves and storms due to these are captured only in $3 \mathrm{c}$ where higher thresholds are set. The stress threshold can only be raised so far: at some point it exceeds the maximum value of stress generated in the model and no lifting can occur. If the lifting rate $\mathrm{R}_{\mathrm{SL}}$ is increased beyond a certain value (dependent on $\tau_{\mathrm{SL}}$ ), unrealistic storms start developing in northern summer from the Acidalia-Chryse region, contrary to observations. The highest stresses in Figure 1 are $\sim 0.06 \mathrm{~Pa}$. Obviously if the stress threshold is larger than this value then there is no dust lifting.

[22] It should be noted, as discussed by Basu et al. [2004], that the specific values of $\mathrm{R}_{\mathrm{SL}}$ and $\tau_{\mathrm{SL}}$ corresponding to a particular dust cycle behavior are specific to the current formulation of the MGCM. The values do show sensitivity to the model horizontal and vertical resolution and also the boundary layer mixing scheme. As the model resolution is increased, the stresses at the lifting centers become higher, hence higher stress thresholds and higher rates of DDL and SL are required for getting the "best fit" simulation. While dust storms occur in the model for a range of $\tau_{\mathrm{SL}}$ that is consistent with laboratory estimated thresholds [Greeley et al., 1992, 2003], it is likely that the values of $\tau_{\mathrm{SL}}$ and $\mathrm{R}_{\mathrm{SL}}$ used in our study compensate for a range of model deficiencies. As such, we believe that if our exact parameterizations were placed in a different GCM, different values of the parameters would be needed to regenerate our results. Put another way, while we have great confidence in the behaviors exhibited by the model and the qualitative accuracy of the parameter space results 
[Basu et al., 2004], we have much less confidence in the actual values of the specific parameters because of known problems with the exact prediction of surface stress values between different numerical models. This likelihood is one of the main reasons to use tunable parameters with atmospheric observations as our guide, rather than using laboratory derived relationships for threshold and injection rate.

\section{Simulated Global Dust Storms}

[23] Several tens of global dust storms have been generated by the GCM for climate states that simultaneously provide a good simulation of the non dust storm atmosphere and strong variability of GDS activity. The simulated global storms can be broadly divided into two categories. The first kind of storms originate from the Hellas basin. These storms can be further divided into two subcategories: early Hellas storms and late Hellas storms. The stress thresholds for such storms lies in the range $0.05-0.058 \mathrm{~Pa}$. The second category includes regional storms triggered by traveling waves in the northern hemisphere.

\subsection{Storms Initiated in the Hellas Basin}

[24] The Hellas basin is a site of strong slope winds and $\mathrm{CO}_{2}$ condensation winds [Silli et al., 1997]. Early-season storms occur in the southwest corner, near the latitude of the retreating polar cap (this site has been commonly seen in other simulations [Newman et al., 2002b; Kahre et al., 2005]. Observations [Banfield et al., 2003] suggest that there is traveling wave activity at this latitude range of southwestern Hellas around the $L_{s}=180^{\circ}$ season. It is likely that this can give the necessary interannual variability to create dust storms. An examination of model results does show a peak in stress variability in this season for this location. Late season storms (starting $\sim L_{s}=240^{\circ}$ and later) occur somewhat further north, starting off from the northern rim of the basin in the latitude range $\left(25^{\circ}-30^{\circ} \mathrm{S}\right)$ of the subtropical westerly jet that develops in the solstice season [Joshi et al., 1995].

[25] The early simulated Hellas storm that starts $\sim L_{s}=$ $195^{\circ}$, spreads eastward due to advection of the lifted dust by the midlatitude westerlies. This is similar to the 2001 global dust storm that also appeared to propagate eastward, triggering lifting centers on the way [Smith et al., 2002; Strausberg et al., 2005]. The simulated storm starts from the southwestern rim of the Hellas basin. Traveling wave activity gives rise to strongest variability of stresses in this part of the basin in the early southern spring season. The storm decays as the westerlies get weaker with approaching summer solstice. Traveling waves also quickly vanish at this time and the polar ice cap no longer lies on the slopes of the Hellas basin. This is also discussed by Newman et al. [2002b]. The storm spreads to most of the southern hemisphere but does not spread effectively in the northern hemisphere. These kind of storms are more regional than global and are weaker in intensity than the late Hellas storms that are truly global in nature. Since the Hadley cell is relatively weak this early in the season, it is not effective in the advection of the dust globally (Figure 2). Together with storms in the northern hemisphere, the early Hellas storm is capable of raising the globally averaged temperatures in the southern spring season to $\sim 200 \mathrm{~K}$ in some simulations (Figure 3c). In the observed 2001 global dust storm there was additional dust lifting from secondary storm centers.

[26] The second kind of Hellas storm that starts around $L_{S}=270^{\circ}$ (Figure $3 \mathrm{~b}$ ) or a little earlier $\left(20-30^{\circ} L_{S}\right.$ earlier) in some cases is much bigger than the early Hellas storms as the intensified Hadley cell circulation in the southern season leads to a rapid distribution of dust. The global dust storm season in the model extends from $L_{s}=230^{\circ}-285^{\circ}$, while smaller storms occur throughout the year [Basu et al., 2004]. Figure $3 \mathrm{~b}$ shows the temperature cycles for our best fit, "variable GDS" interannual simulation. This model readily generates a range of GDS sizes and initiation dates within the "dust storm season", and yields peak air temperatures which are quite close to those observed. The individual storms within this simulation can be examined more closely and compared with spatially resolved data. These storms originate mostly from the northern rim of the Hellas basin where the subtropical jet helps in raising the stresses. Stresses from the jet are tied to the strength of the Hadley cell circulation. In some years there are two storms, in some years there is only one big storm and these are interspersed by no storm years (Figure 3). Haberle et al. [1993] and Murphy et al. [1995] show that there is a strong positive feedback effect at play. An intensified Hadley circulation leads to a stronger subtropical jet. This likely leads to lifting at other longitudes along this latitude. This storm spreads along the Hadley cell convergence zone. This occurs along a zonal collar at latitude $\sim 30^{\circ} \mathrm{S}$ in the beginning and then spreads out in all directions. When the Hadley cell circulation becomes strong enough, it activates a secondary lifting center along the same zonal collar near Argyre. This secondary lifting center helps in making the storm truly global. The years that the secondary lifting center is not activated, the storm is contained as a local Hellas storm and does not become a global storm. The storm decays as the Hadley cell circulation becomes less intense toward the end of southern summer. The trend in peak opacity results from the fact that the modelled GDSs do not "switch-off" properly. This issue is discussed further in section 6. The spread of GDS initiation date compares well with the historical record [Martin and Zurek, 1993]. GDS events are limited to southern spring and summer, with a bias toward mid-to-late southern spring. This bias can also be seen in Figure 3, where the predicted global-mean, midlevel air temperatures are compared with observations of Martian storms.

[27] Figure 5 provides a summary of the initiation season versus peak opacity for these late season global dust storms that are initiated from the northern rim of Hellas with the help of the subtropical jet. The earlier the storm is initiated, the greater its intensity and it reaches higher peak opacities. This is illustrative of the positive radiative dynamical feedback. The earlier the dust gets entrained in the Hadley cell circulation, the more time the Hadley cell gets to develop and the bigger the storm gets.

[28] The observed 2001 global dust storm began quite early, just after equinox $\left(L_{s}=185^{\circ}\right)$, so that the southern spring and summer Hadley circulation is only just established and is very weak. However, this storm became global since it was more like a cascade of storms helped by additional lifting centers like Solis Planum and Daedalia 


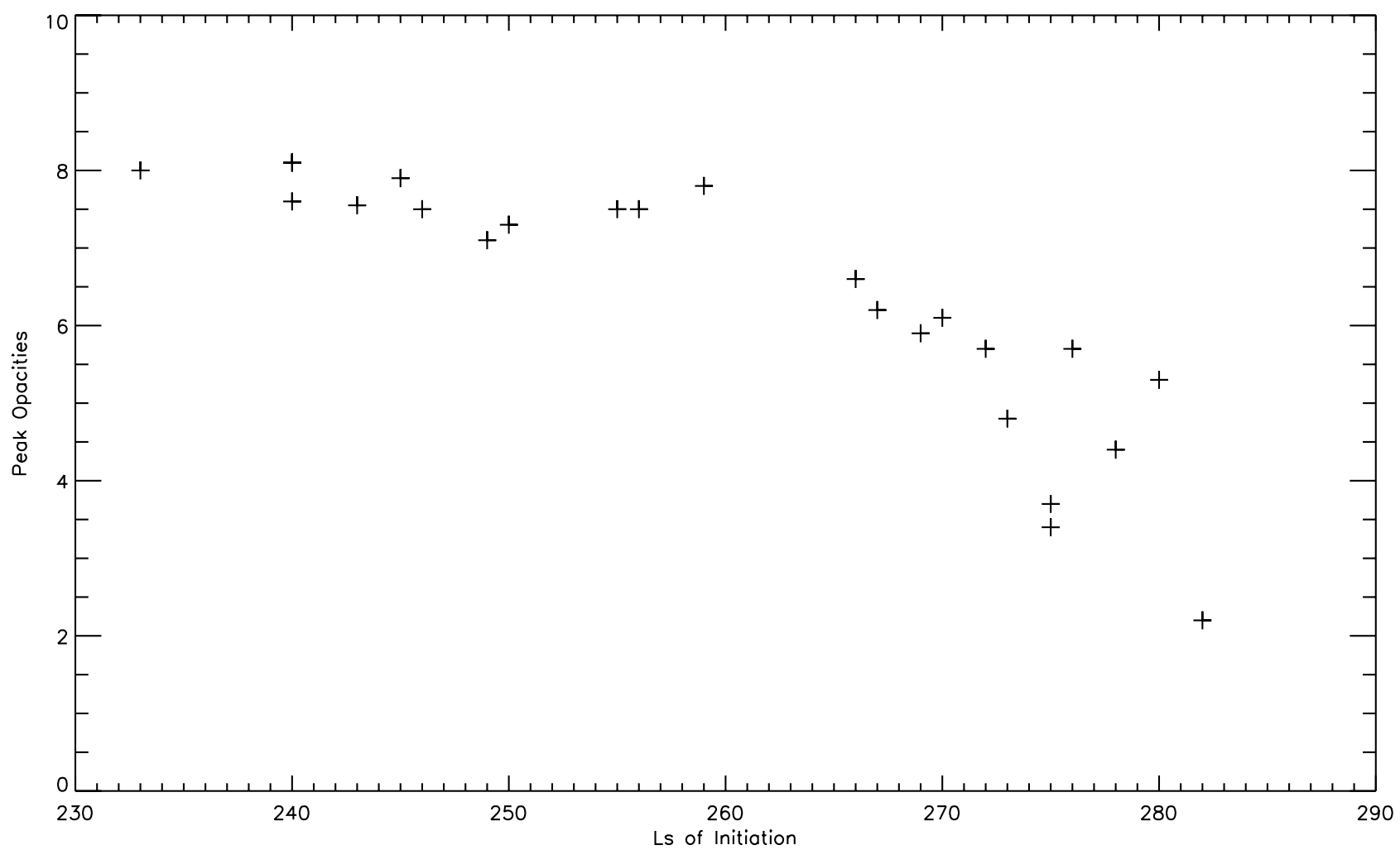

Figure 5. Plot of initiation time $\left(L_{s}\right)$ versus peak opacity for the "best fit" variable global dust storms. Storms that originate earlier in the season reach higher peak opacities than the ones that originate later.

rather than a single Hellas storm. The GCM-predicted window for GDS development overlaps substantially with, but is somewhat smaller than, that observed.

\subsubsection{Evolution}

[29] The general evolution of an early Hellas storm as described in section 4.1 is shown in Figure 6. The dust opacity has been normalized to remove the influence of topography. The storm starts from the southwestern rim of Hellas $\sim L_{s}=193^{\circ}$ and subsequently fills the whole basin and starts spreading eastward from the southeastern rim of Hellas into Eridania and Sirenum. This is similar to the 2001 GDS. An independent storm event in the Alba Patera region can be seen in the northern hemisphere at $L_{S}=202^{\circ}$. In some instances, such events in Alba Patera and the Chryse region give rise to cross-equatorial storms that, together with the Hellas storm, fill most of the planet and raise the global average temperatures to $\sim 200 \mathrm{~K}$ during southern hemisphere spring season. The storm decays $\sim L_{s}=$ $205^{\circ}$ when the westerlies become weaker.

[30] The evolution of one of the global dust storms beginning at roughly $L_{s}=240^{\circ}$ can be seen in Figure 7 . This figure shows the geographic distribution of column opacity at six time intervals covering the storm evolution. The dust opacity has been normalized to remove the influence of topography. The net lifting/deposition of dust per day for each of the six time steps is also shown. The storm begins on the northwestern rim of the Hellas basin. By the first time frame, dust is beginning to "probe" to the north and to the east of Hellas. We use the word "probe" to convey dust transport which is not smooth, but instead reflects the pulsating (advective) transport associated with the strong modulation of the winds by the diurnal thermal tide, and dissipation of dust pockets advected from Hellas and cut off by the tidal reversal of the winds. The northward transport moves dust into Syrtis Major, while the eastward transport moves dust into Hesperia. In Figure $7 \mathrm{a}$ and $7 \mathrm{~b}$, one can also make out a secondary dust-lifting center along the seasonal cap edge to the south and west of Tharsis. The early evolution of the storm, including initiation in Hellas, transport to the north and east within distinct fingers, and the activation of lifting along the southern cap edge south of Tharsis all provide strong echos of the actual development of the 2001 GDS. We must be careful, as that storm began much earlier than our $L_{s}=240^{\circ}$ event, and the subsequent evolution of the modeled storm and the 2001 Mars GDS differ.

[31] By the next frame $\left(L_{s}=251^{\circ}\right.$, Figure 7c), the dust has encircled the planet at high southern latitudes. The dust is advected both eastward and westward. The easterly winds are stronger in this season and play an important role in the advection of dust. Dust lifting along the cap boundary also helps spread the dust around the planet. The northern latitudes also experience increased opacities, but not to the same degree: the dust distribution is strongly biased to the south since that is where the lifting is taking place. The majority of the dust lifting remains concentrated on the northern rim of Hellas (Figure 7d). Additional lifting occurs along the southern seasonal $\mathrm{CO}_{2}$ ice cap edge. A local peak in lifting can be seen to the south and east of Tharsis. Comparing Figures $7 \mathrm{~d}$ and $7 \mathrm{~b}$, it can been seen that net lifting outside of these major lifting regions is diminishing and turning into net deposition in many areas. This repre- 

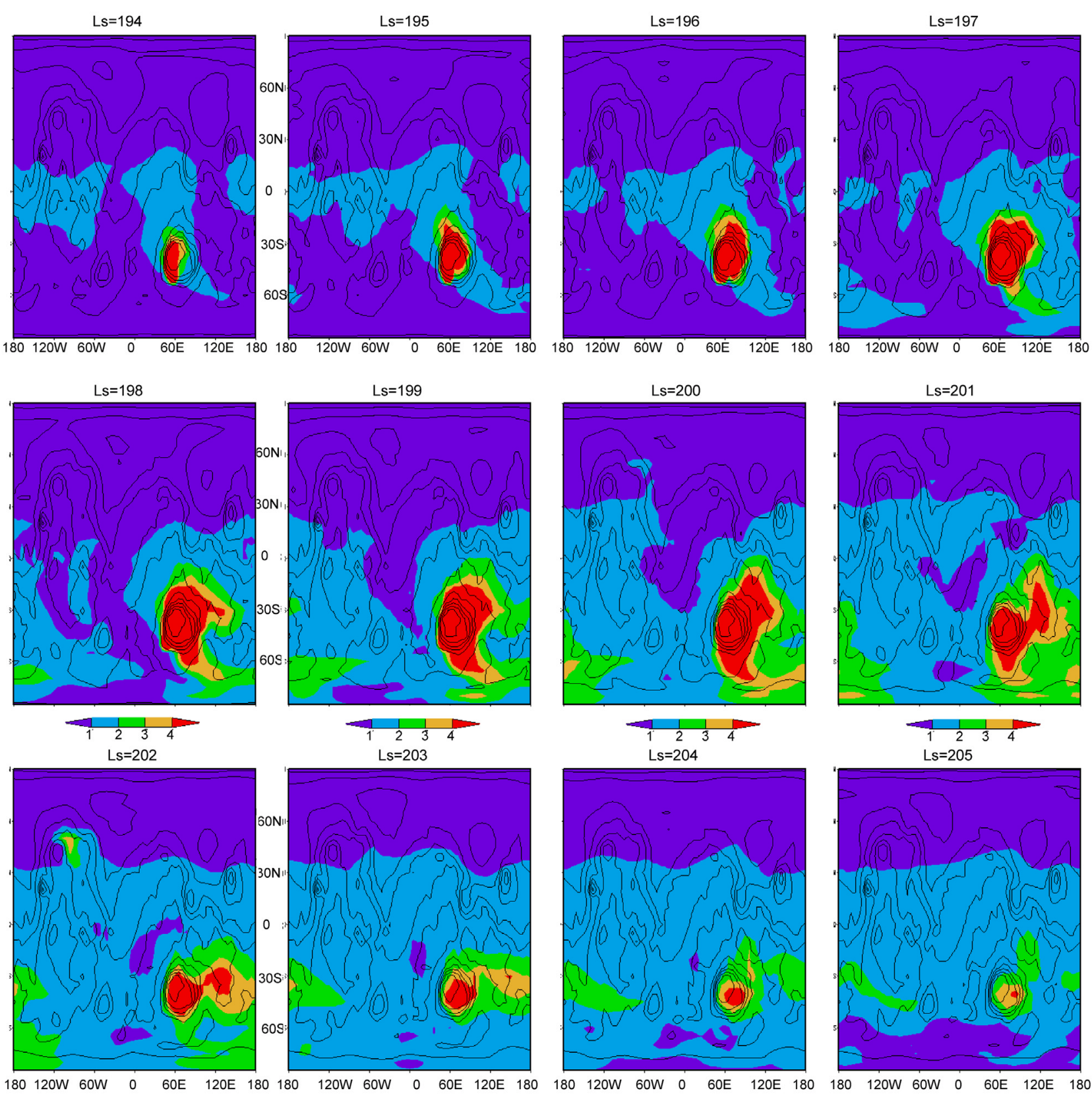

Figure 6. Geographic distribution of normalized opacity (to $6 \mathrm{mb}$ ) at 12 time intervals covering the storm evolution. The storm starts $\sim L_{s}=195^{\circ}$. An independent storm just north of Alba Paterra can also be been at $L_{s}=202^{\circ}$. The globally averaged $T_{15}$ cycle for this simulation is shown in Figure 3c (year 2). This is a good example of a simulated early Hellas storm.

sents some reduction in dust lifting by dust devils, but is largely due to significant increase in dust fallout associated with the higher atmospheric dust opacity.

[32] The following ten degrees of $L_{s}$ (from $L_{s}=250^{\circ}-$ $260^{\circ}$, Figures $7 \mathrm{e}-7 \mathrm{~h}$ ) see the circulation exporting much more dust to the northern hemisphere, with opacities exceeding 2 everywhere south of roughly $40^{\circ} \mathrm{N}$ by $L_{s}=260^{\circ}$. In fact, while there is substantial change in the storm between Figures 7a, 7c, and 7e, there is much more muted change between Figures $7 \mathrm{e}$ and $7 \mathrm{~g}$. Figure $7 \mathrm{~g}$ corresponds to $L_{S}=260^{\circ}$, or roughly $20^{\circ}$ of $L_{S}$ after storm initiation. This is roughly the interval after which the 2001 GDS began to decay. (Actually, the peak opacities of the 2001 storm begin decaying around $L_{s}=215^{\circ}$ [Smith et al., 2002; Strausberg et al., 2005].) However, the simulated storm does not decay (see section 5), as dust lifting continues vigorously. Figures $7 \mathrm{f}$ and $7 \mathrm{~h}$ show that the lifting on the northern rim of Hellas and southeast of Tharsis has increased relative to the state in Figure $7 d$. Only a very small fraction of the planet exhibits net injection of dust by this point, being overwhelmed by dust sedimention, as mentioned above. Observations of the 2001 global storm suggest that the lifting center on the northern rim of Hellas switched off after the secondary lifting center southeast of Tharsis initiated 

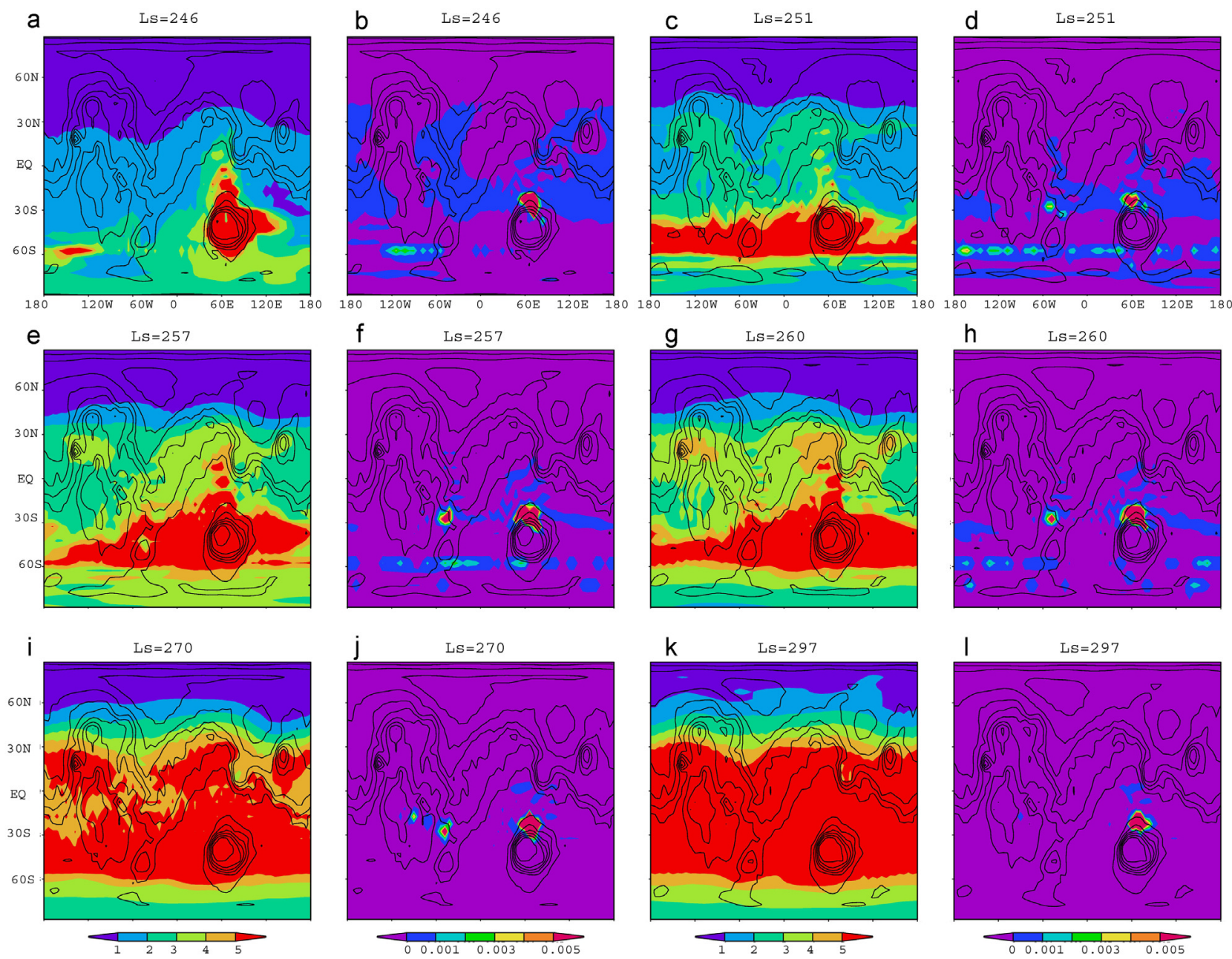

Figure 7. (a, c, e, g, i, k) Geographic distribution of normalized opacity (to $6 \mathrm{mb}$ ) at six time intervals covering the Hellas storm evolution. (b, d, f, h, j, l) The net dust lifted $\left(\mathrm{gm} / \mathrm{cm}^{2}\right)$ per day for the corresponding six time intervals is also shown in alternate panels. These give an idea of the dust-lifting centers at those particular $L_{s}$. The storm begins from the northwestern rim of the Hellas basin. It spreads into Syrtis Major in the north and toward Hesperia in the east. The secondary dust-lifting center can be seen along the southern cap edge south of Tharsis. The topography is contoured in black. The globally averaged $T_{15}$ cycle for this simulation is shown in Figure $3 \mathrm{~b}$ (year 3 ). This year has one of the biggest simulated storms.

[Strausberg et al., 2005]. This is not happening in this simulation and both centers remain active until roughly $L_{s}=$ $260^{\circ}-270^{\circ}$. Indeed, even at $L_{s}=297^{\circ}$, the Hellas northern rim lifting center remains very active (Figure 71). This steady injection of dust allows the model to produce a very smooth distribution of opacity (i.e., a relatively uniformly mixed aerosol distribution) in the final two time steps of Figure 7, corresponding to $L_{s}=270^{\circ}$ and $297^{\circ}$. As can be seen in Figure $3 \mathrm{~b}$, the storm decays only after roughly $L_{s}=$ $300^{\circ}$.

[33] We also simulate some small local dust storms in the Hellas region in northern spring and summer. In reality these storms may be supressed somewhat by the effects of water ice cloud formation on dust nuclei. Observations show Hellas to be filled with water ice clouds in this season [Wang and Ingersoll, 2002] Currently, we are not representing the possible scavenging and radiative impact of water ice clouds. These storms are trigerred in the model along the rims of Hellas when the transient stresses have a seasonal maximum.

\subsubsection{Dust and Air Temperature Distributions}

[34] The latitudinal distribution of mid-level air temperature provides additional information on the dynamical response of the atmosphere during a GDS. In Figure 8, we present synthetic $T_{15}$ temperatures derived from the GCM simulation shown in Figure 3c, year 1 alongside data from the 1977 dust storm season. The $T_{15}$ values are synthesized from the model through application of the IRTM $15-\mu \mathrm{m}$ channel weighting function [Wilson and Richardson, 2000]. The IRTM data are not systematically sampled and contain nonrandom changes in observation local time, associated with drift in the orbits of the Viking Orbiters. The IRTM tropical temperatures are biased by the local time sampling, which favors local times when the 

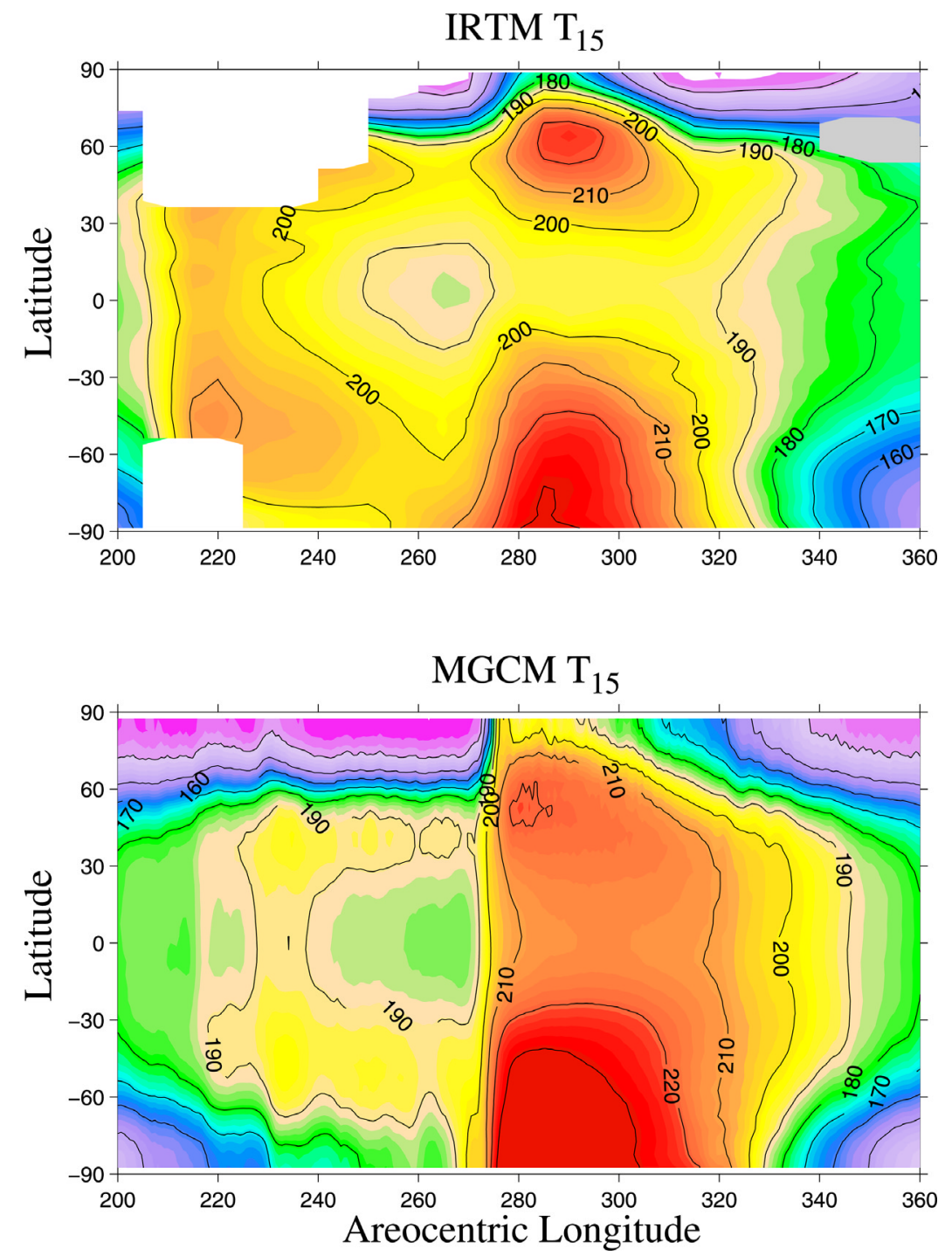

Figure 8. Synthetic IRTM $T_{15}$ data (K) derived from a GCM simulation that has a late Hellas storm $\sim L_{s}=280^{\circ}$ (Figure 3c, year 1) along with data from the 1977 dust storm season. The southern spring and summer season is shown for each.
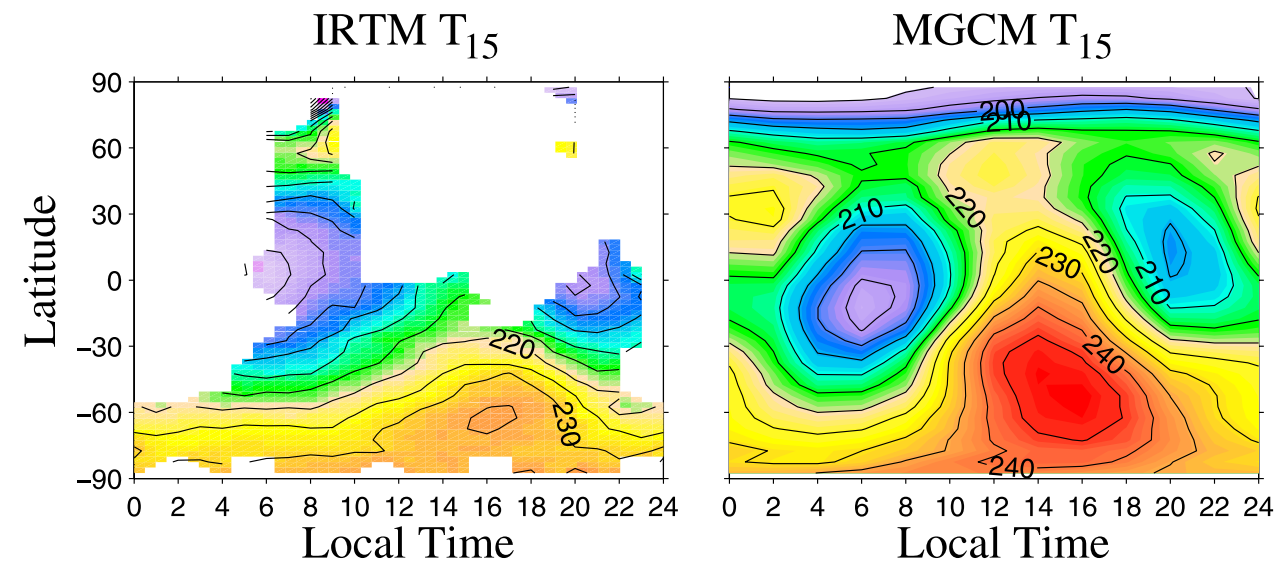

Figure 9. The diurnal variation of simulated $T_{15}(\mathrm{~K})$ at the peak of the solstitial dust storm shown in Figure $3 \mathrm{c}$, year 1 compared to the $1977 \mathrm{~b}$ dust storm data. The diurnal variation is derived from a 10-day segment of model output, with temperatures composited every 2 hours. 


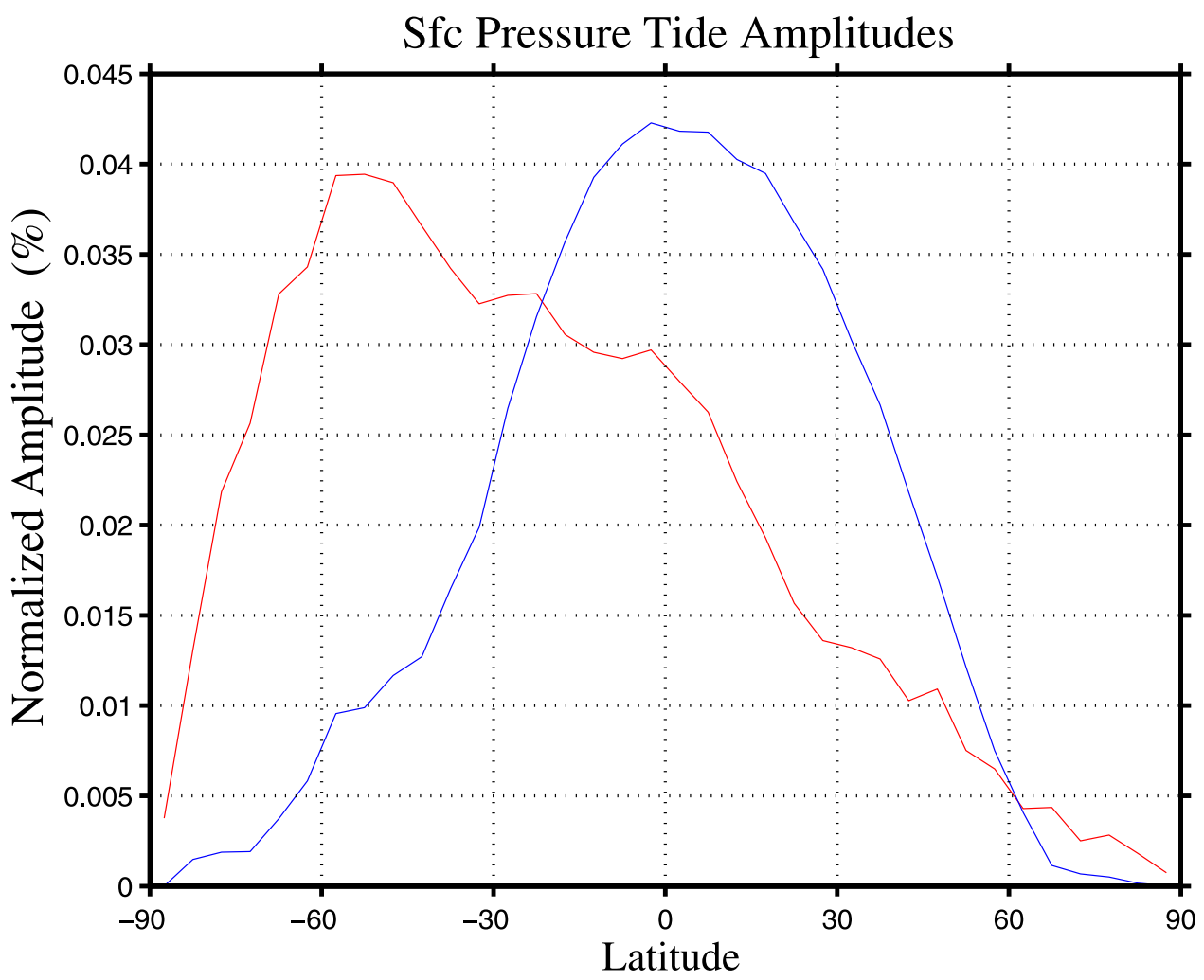

Figure 10. The simulated diurnal (red) and semidiurnal (blue) tide components (the migrating tides) at $L_{S}=280^{\circ}$ for a year that has a global dust storm (Figure $3 \mathrm{c}$, year 1 ). The amplitudes at $22^{\circ} \mathrm{N}$ latitude and $48^{\circ} \mathrm{N}$ latitude are in very good agreement with those observed at the Viking Lander 1 and Viking Lander 2 sites at the peak of the 1977 b global dust storm.

strong semidiurnal tide yields minimum temperatures (Figure 9).

[35] As the storm decays (Figure 8), the simulation and the observations are characterized by a strong, doublepeaked latitudinal distribution of temperature. The peaks are in the midlatitudes of both hemispheres. The southern peak corresponds to direct heating, while the northern peak is a result of adiabatic warming associated with the descending branch of the Hadley cell. Both the model and the observations show a trend of poleward motion of the temperature peaks during this period.

[36] The $1977 \mathrm{a}$ dust storm began around $L_{s}=205^{\circ}$. There is a smaller regional Hellas storm in the GCM simulation that starts $\sim L_{s}=220^{\circ}$ (Figure 8). The IRTM data and the model are roughly similar at $L_{s}=270^{\circ}$, just prior to the start of the $1977 \mathrm{~b}$ storm. The model has less cap-edge dust lifting in the southern hemisphere, so the simulated polar temps in the southern hemisphere and at $60^{\circ} \mathrm{N}$ are on the cold side. The strong dust heating in the southern hemisphere after the simulated storm development leads to winter polar warming. This warming lasts longer in the model due to slower storm decay. The mechanism of this polar warming is described by Wilson [1997] and Forget et al. [1999]. This warm pole bias in the model dissipates with the spin-down of the Hadley cell by roughly $L_{s}=320^{\circ}$. Interestingly, the decay of the model storm in terms of rate and trend in latitudinal distribution, agree rather well with observations of the $1977 \mathrm{~b}$ storm. The southward drift of peak northern hemisphere temperatures between $L_{s}=285^{\circ}$ and $320^{\circ}$ is particularly well captured. In short, while there are some differences, the model does extremely well at emulating the observed seasonal variation of meridionally resolved, midlevel air temperatures before, after, and during a major dust storm. Once dust lifting has ceased, the simulated storm decay is a strong function of dust particle sedimentation. Of course, the season will be relevant as well, since the Hadley circulation can be more or less effective at keeping dust lofted in the tropics.

[37] Comparison of tide amplitudes between simulations and data provides a measure of the dust heating in the simulation. Figure 9 shows the diurnal variation of the simulated $T_{15}$ temperatures at the peak of the solstitial dust storm shown in Figure 8. It is based on a 10-day segment of data, with temperatures composited every 2 hours. There is a strong semidiurnal tide in the tropics and a diurnal tide in the summer midlatitudes. In general, temperatures are warmer than the IRTM temperatures and the semidiurnal tide amplitude is stronger. These suggest that dust heating is stronger in the simulation than observed. Qualitatively, the correspondence is good. Also as discussed earlier, it is important to keep in mind the sampling bias in the IRTM temperature observations. The amplitudes of surface pressure tides have a better correspondence when compared with Viking Lander observations. Figure 10 shows the simulated diurnal (red) and semidiurnal (blue) tide components or the migrating tides. The semidiurnal tide is an excellent measure of global heating [Zurek, 1981]. Its amplitudes at $22^{\circ} \mathrm{N}$ latitude and $48^{\circ} \mathrm{N}$ latitude are in very 

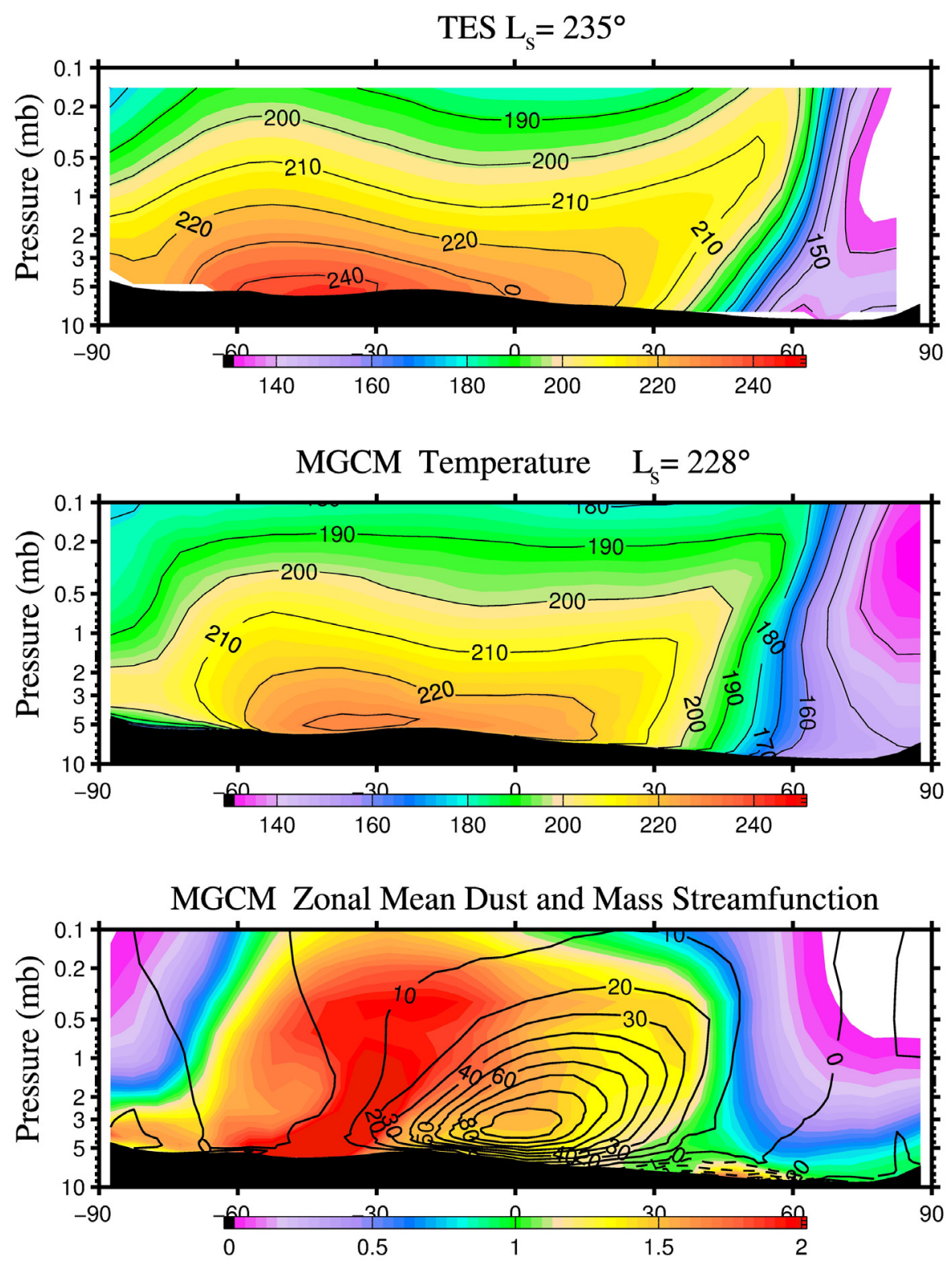

Figure 11. Comparison of zonal mean TES temperatures (K) (from the first mapping year) and MGCM diurnally averaged zonal mean temperature (Figure 3c, year 2). The dustiness and temperatures maximized at this time following the Chryse dust activity. Note that TES is $0.5 *\left(\mathrm{~T}_{2 \mathrm{am}}+\mathrm{T}_{2 \mathrm{pm}}\right)$, compared to MGCM which is true zonal and diurnal average. The MGCM storm peaks at $L_{s}=228^{\circ}$, whereas the observed storm peaks at $L_{s}=235^{\circ}$. The third panel shows the zonal mean dust opacity (shaded) and mass stream function (contoured) for the MGCM simulation at $L_{s}=228$. The units of mass stream function are in $10^{8} \mathrm{~kg} / \mathrm{s}$. Solid contours show clockwise circulation.

good agreement with those observed at the Viking Lander 1 and Viking Lander 2 sites at the peak of the 1977b global dust storm. This suggests that we roughly have the right amount of globally integrated dust heating in the simulation.

[38] Latitudinal cross sections of temperature and dust in the simulations and data give us an additional means of investigating the similarities and differences between the model and the actual atmosphere. We compare the temperature profiles as a function of height and latitude for our simulations and TES observations from the first mapping year (Figure 11). These are compared for $\sim L_{s}=230^{\circ}$ when both reach peak temperatures during a Hellas storm. In TES observations this storm began as a sequence of flushing events [Smith, 2004]. This is the early Hellas storm in the simulation that we discussed in section 4.1. The northern hemisphere warming is stronger in TES data, which is suggestive of a stronger Hadley cell circulation. The MGCM is warmer at $50^{\circ}-60^{\circ} \mathrm{N}$ near the surface. The correspondence between the two latitudinal cross sections is quite good, considering the fact that high latitude temperatures in the southern hemisphere will increase with the evolving season, as the subsolar latitude moves southward 

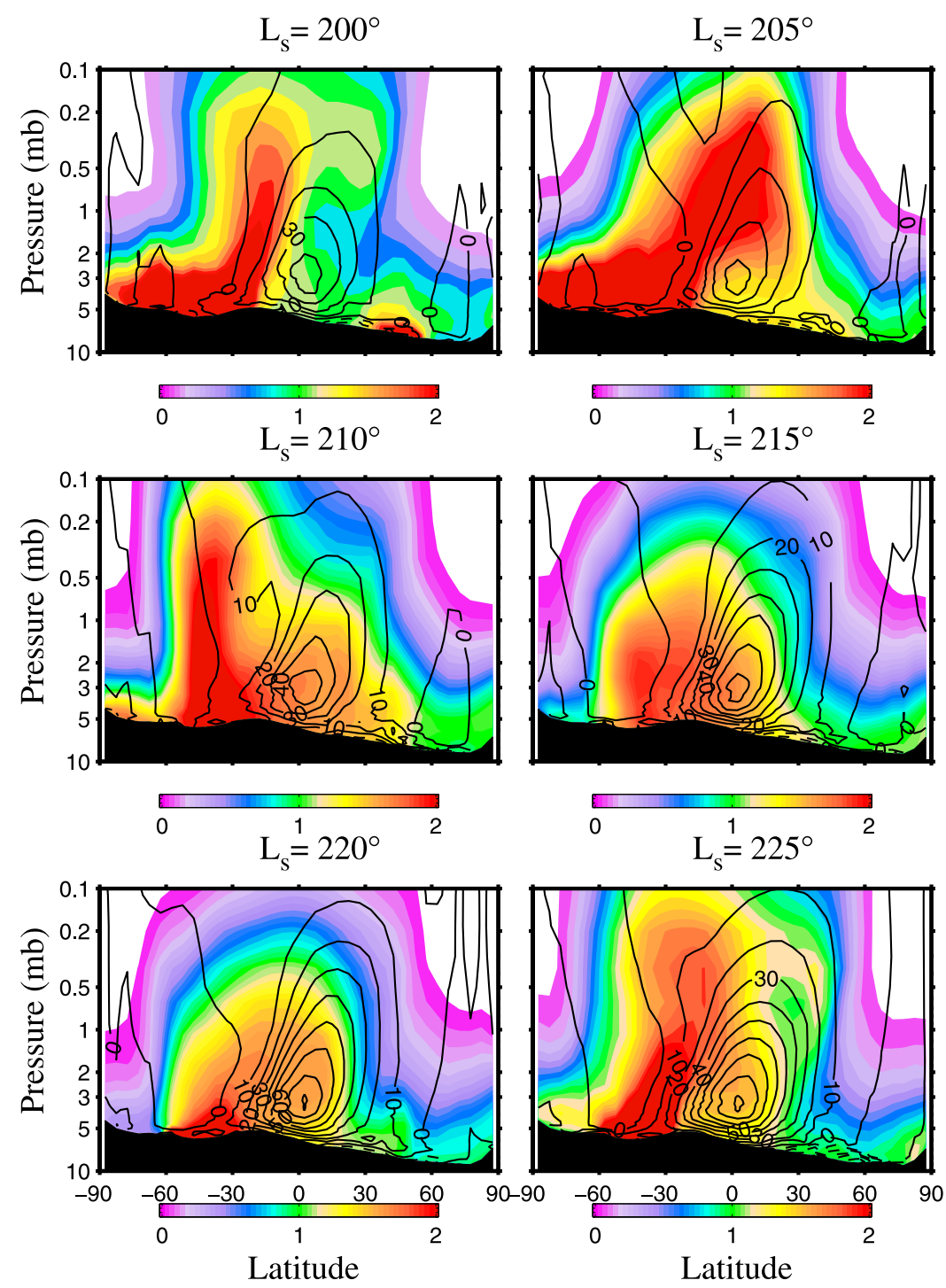

Figure 12. Vertical dust distribution (shaded) and mass stream functions (contoured) are plotted for a simulated early Hellas storm (Figure 3c, year 2). In this year of simulation, there were two temperature peaks: one at $L_{s}=205^{\circ}$ and one at $L_{s}=228^{\circ}$. Note that at $L_{s}=200^{\circ}$ there is Chryse storm activity in the northern hemisphere, which is much weaker than the Hellas storm. It is located in the descending branch of the Hadley circulation. The convention for the contour lines is solid lines show positive stream functions $\left(10^{8} \mathrm{~kg} / \mathrm{s}\right)$ or clockwise circulation.

in the simulation. TES southern polar temperatures are warmer, especially in the higher levels of the atmosphere, as it is later in the season $\left(L_{s}=235^{\circ}\right.$ as opposed to $L_{s}=228^{\circ}$ for the simulation) when atmospheric temperatures and thermal tides maximize.

[39] Figure 12 shows a simulation that has early Hellas storms starting at $\sim L_{s}=195^{\circ}$. This gets entrained into the Hadley cell circulation by $\sim L_{s}=203^{\circ}$ and subsequently settles out $\sim L_{s}=219^{\circ}$. The Hadley cell is not very strong at this time; hence the storm is not very strong either. A second storm peaks at $\sim L_{s}=228^{\circ}$ (not shown in Figure 12). Chryse storm activity is also simulated in the northern hemisphere $\sim L_{s}=200^{\circ}$. These baroclinically raised dust events intensify moderately as they travel southwards, but still are much weaker than the Hellas storm. There is interannual variability in the simulation in terms of the intensity and timing of the early Hellas storms as well as the Chryse storms. However, the dust lifting in the northern hemisphere is insufficient compared to observed storms.

[40] Note that the Hadley cell circulation is intensifying rapidly as the season evolves away from equinox toward southern summer solstice. This plays a more important role than dust, for this storm. The figure also shows the mass transport stream function in this season is much more tropically confined than in the solstice seasons [Haberle et al., 1993; Wilson, 1997; Forget et al., 1999], with the strong upward branch centered near the subsolar latitude, just south of the equator. The maximum stream function value $(\sim 50 \times$ 

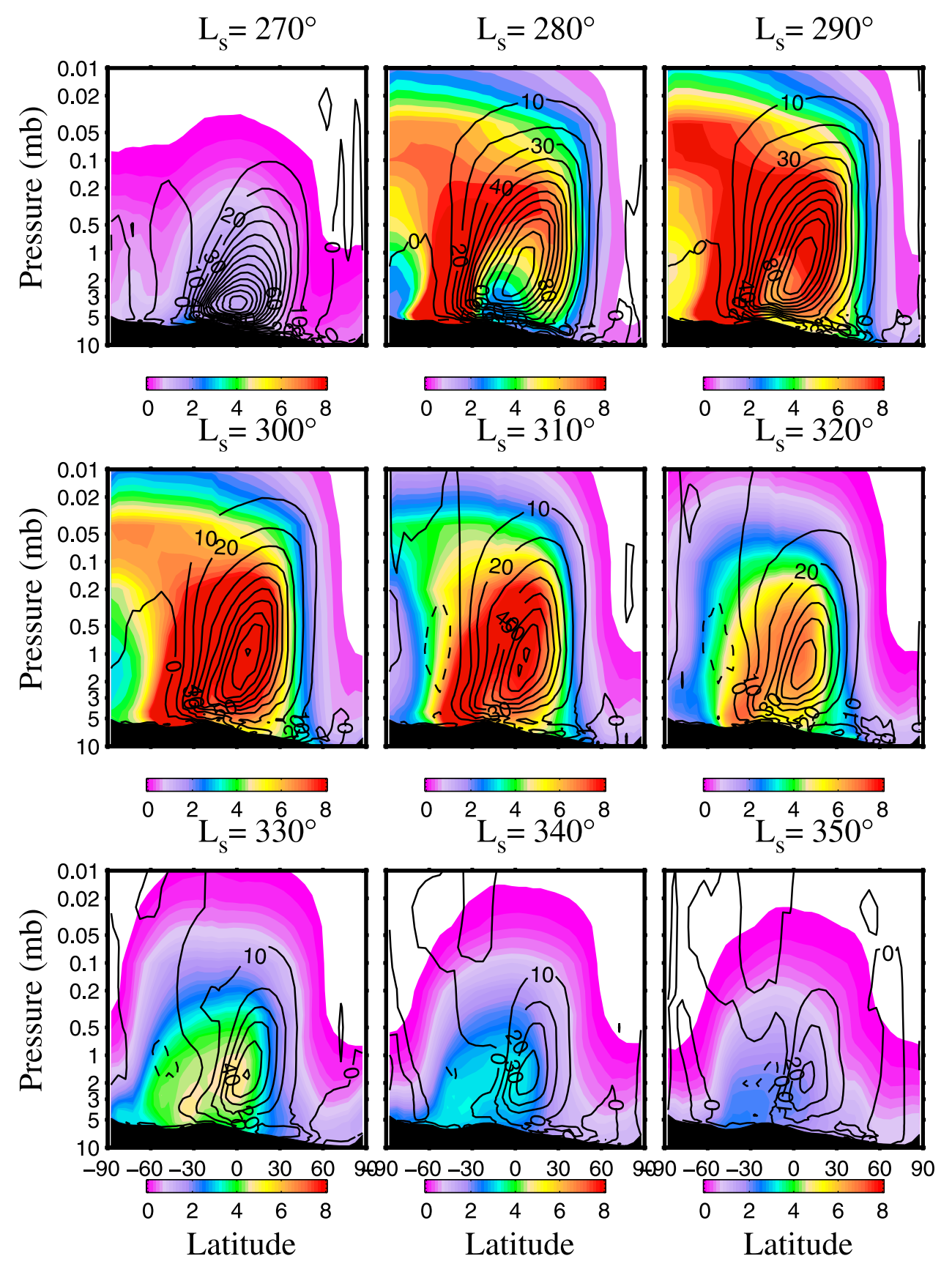

Figure 13. Dust opacity evolution for simulated $L_{s}=270^{\circ}$ storm (Figure 3c, year 1 ). The shadings and contours are the same as Figure 12. The storm peaks at $\sim L_{s}=300^{\circ}$ and decays as the Hadley cell circulation becomes weaker.

$10^{8} \mathrm{~kg} / \mathrm{s}$ ) is significantly weaker than seen in simulations of the southern hemisphere summer solstice case, where values range from $100-15010^{8} \mathrm{~kg} / \mathrm{s}$. The simulated dust distribution in Figure 12 is clearly influenced by the axisymmetric circulation, with maximum vertical extent in the rising branch of the Hadley circulation. The intense downward branch of the Hadley circulation significantly limits the poleward extent of dust in the northern hemisphere. This adiabatic descent accounts for the relatively warm temperatures in the northern hemisphere. The model indicates a low level leakage of dust into higher northern latitudes, which is attributable to stationary and traveling waves, as this behavior is not seen in axisymmetric model calculations. Similarly, dust is confined to relatively low altitudes in the southern hemisphere polar region. The pattern of the dust distribution and atmospheric circulation is quite robust, and is not dependent on the details of the source specification or dust-lifting rates.

[41] This sequence of panels shows strong initial transport of dust to high southern latitudes, although not to high altitudes. This could be due to planetary waves. Dust is transported to high altitudes in the tropical region. High 

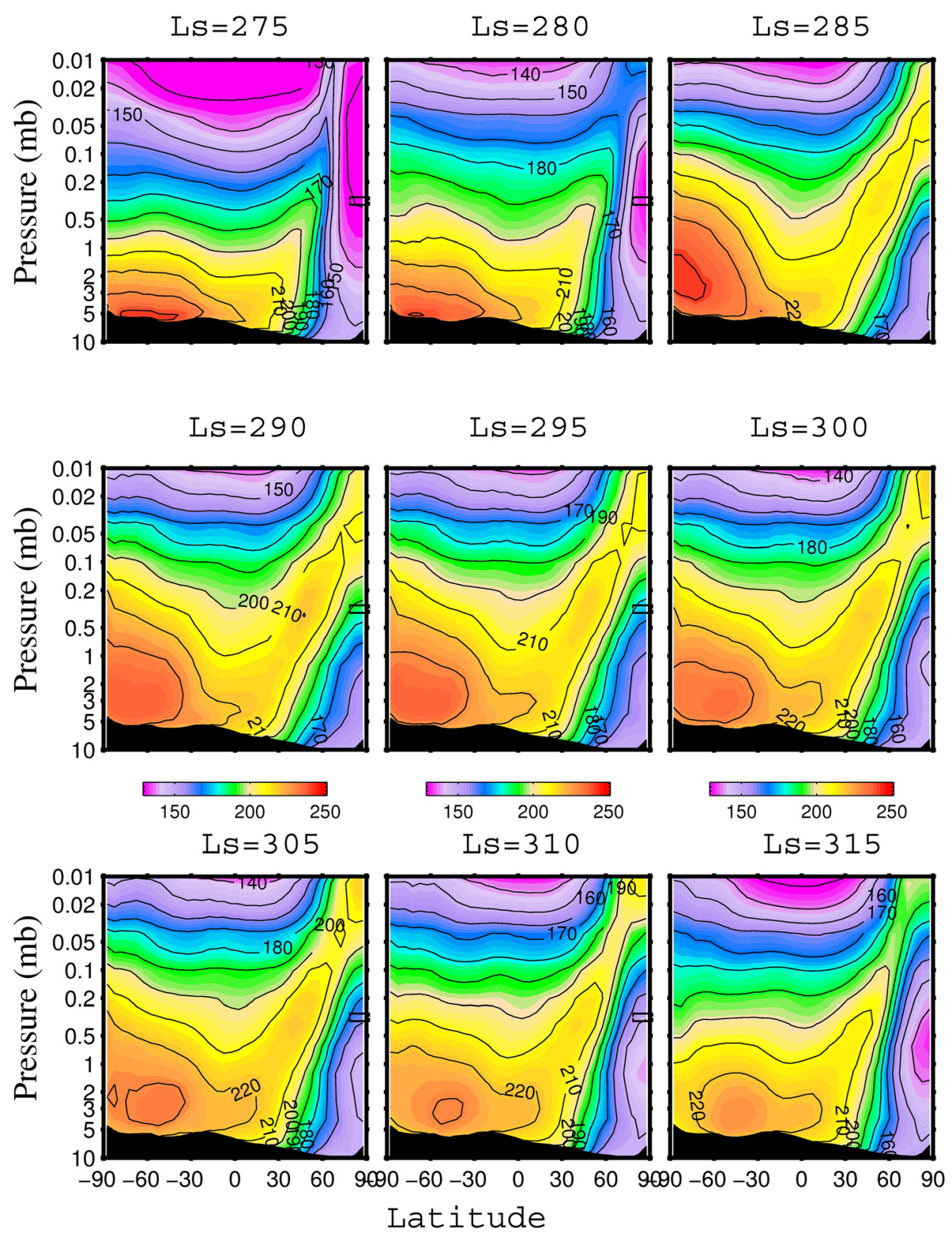

Figure 14. Latitude-height section of zonally averaged temperatures $(\mathrm{K})$ for dust storm evolution (Figure 3c, year 1). The dust storm starts out in Hellas basin, where the dust is lifted into lower levels of the atmosphere. It spreads into the northern hemisphere predominantly through higher levels in the atmosphere $\sim 25 \mathrm{~km}$. The explosive development of the global dust storm occurs when dust lifting in Hellas is sufficient to intensify the Hadley cell circulation and wind stresses in a portion of the southern tropical convergence zone exceed $\tau_{\mathrm{SL}}$. This activates the secondary lifting centers, and the dust storm becomes global.

altitude transport has been simulated in the solstice season by Wilson [1997] and Newman et al. [2002a, 2002b]. These have been attributed to tidal activity. Following the dustlifting episode, dust tends to be confined to the tropical regions.

[42] The dust and temperature cross sections for the late Hellas storm that starts $\sim L_{s}=270^{\circ}$ are shown in Figure 13 and Figure 14, respectively. It can be seen that the Hadley cell circulation is quite intense between $L_{s}=270^{\circ}-300^{\circ}$ after which it weakens and so does the dust storm. The maximum stream function value $\left(\sim 100 \times 10^{8} \mathrm{~kg} / \mathrm{s}\right)$ is significantly stronger than seen in simulations of the presolstice storms (Figure 12). The signature of a strong source of dust from the Hellas region is present until $L_{s}=320^{\circ}$, when the dust distribution relaxes back toward being relatively uniformly mixed. Even though the peak opacities are reached at $\sim L_{s}=295^{\circ}$, the source does not shut down till $L_{s}=320^{\circ}$ when the Hadley cell circulation also decays. Thus the high opacities are maintained for a long time. There is major northern hemisphere polar warming in this 


\section{V-Shaped Chryse Storm}

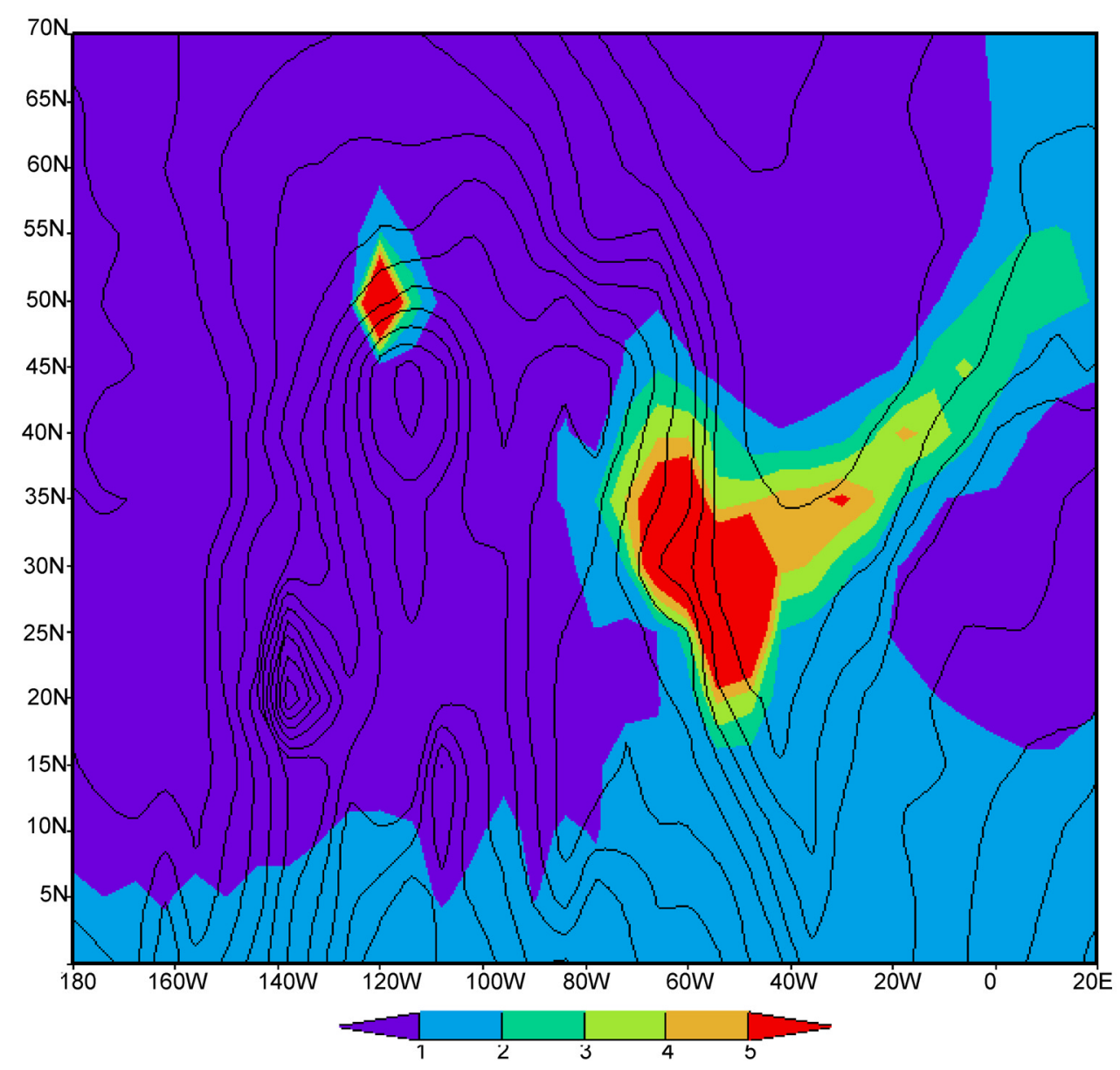

Figure 15. Spatial plot of normalized dust opacity showing the development of a V-shaped storm in the Acidalia-Chryse region (Figure 3c, year 1). The black contours show the topography.

simulation. The distribution of dust, with advection over the South Pole at high altitude is similar to that shown by Wilson [1997] for the 1977b storm.

\subsection{A Regional Dust Storm Initiated by a Northern Hemisphere Baroclinic Storm}

[43] While most global storms generated by the model initiate in or near the Hellas basin, smaller storm events are triggered and evolve in different ways in the northern hemisphere. There is frequent dust lifting here, just not enough to be really noticeable. Most of these events trigger in the Acidalia region and are subsequently propagated eastward and southward into the Chryse basin. The other lifting centers in the northern hemisphere are Alba Patera, Amazonis, Olympus Mons, Arcadia, Acidalia/Chryse, Syrtis Major, Isidis and Elysium Mons. Most of these are activated by the Chryse storm and are independent of the storm originating from the Hellas region.

[44] There are many events in which dust lifting initiates in the northern (autumnal) hemisphere. The dust opacity distribution for one such storm is seen in Figure 15. The $\mathrm{V}$ shape that the storm assumes has also been observed by TES [Wang et al., 2003, 2005]. This particular storm starts $\sim L_{s}=220^{\circ}$ and develops into a cross-equatorial flushing storm.

[45] The intensity and spatial extent of the Chryse storms are possibly affected by the latitudinal extent of the seasonal ice caps in the northern hemisphere. Experiments that were run with lower obliquities produced much bigger Chryse storms that went global in some cases. The extent of the seasonal ice caps in the northern hemisphere is less than a higher obliquity case and this is likely to affect the intensity of the traveling waves and hence the intensity of the Chryse storms. The other possibility is that the Hellas storms are weaker in lower obliquity simulations due to a weaker subtropical jet. The southern hemisphere ice cap is also further away from the Hellas slopes thus reducing the transience in the Hellas basin circulation at $L_{s} \sim 180^{\circ}$. A weaker Hellas storm does not overwhelm the circulation in the northern hemisphere and thus the Chryse storm has the opportunity to develop into a full-fledged storm.

[46] The evolution of dust opacity and dust-lifting centers from such an intensified storm is illustrated in Figure 16, paralleling the presentation for a Hellas dust storm in Figure 7. The storm begins in mid-northern autumn (somewhat before $L_{s}=220^{\circ}$, Figure 16a), at the northeastern edge 

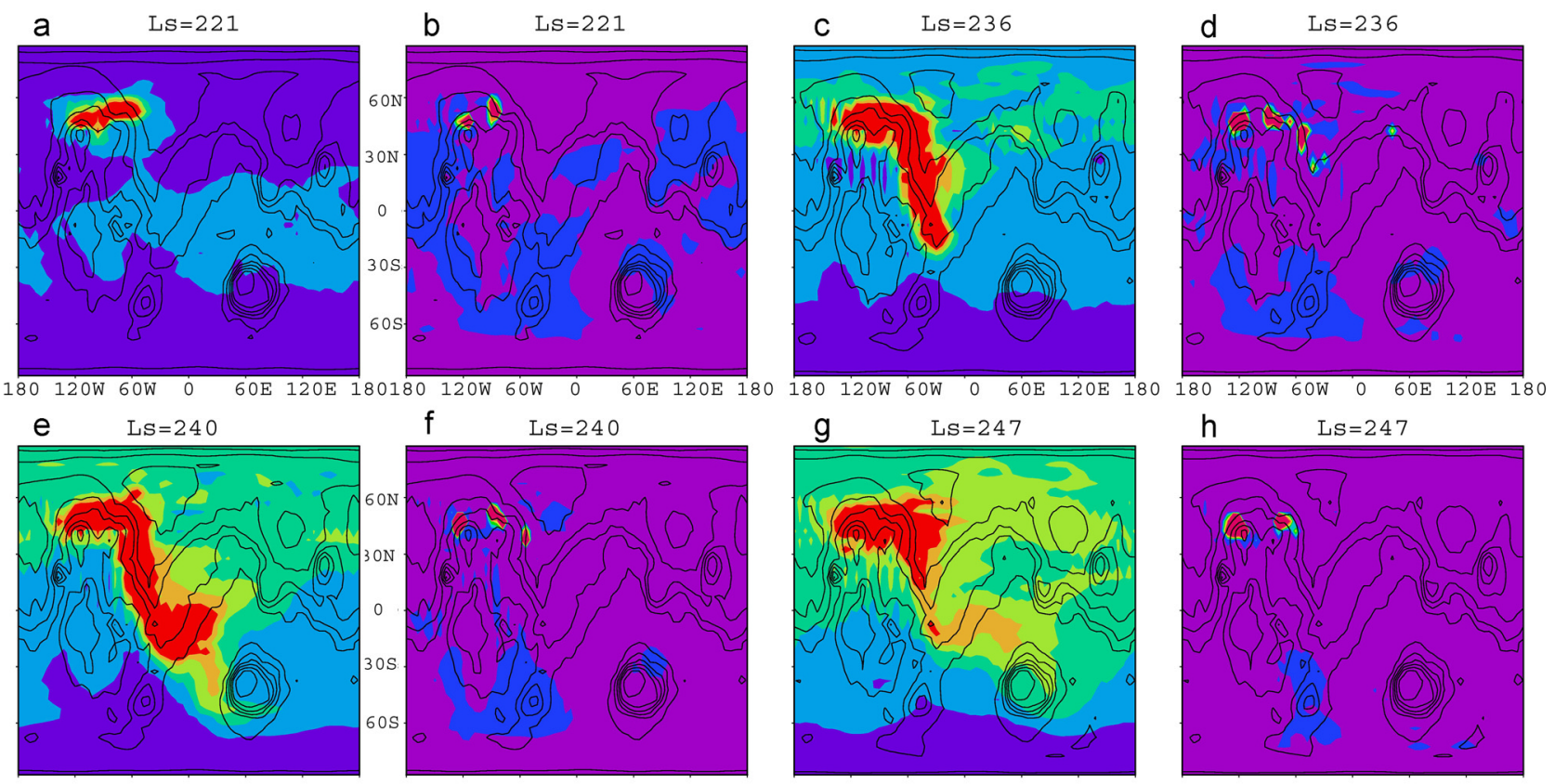

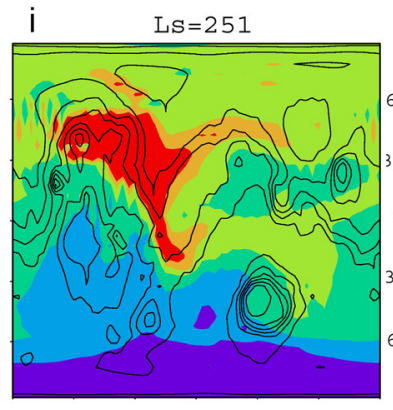

\begin{tabular}{l|lllll} 
& & & & & \\
1 & 2 & 3 & 4 & 5
\end{tabular}

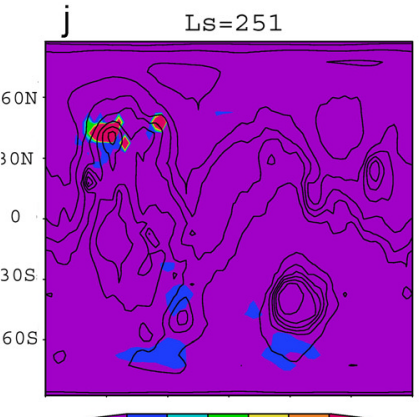

$\begin{array}{llll} & 0.001 & 0.003 & 0.005\end{array}$
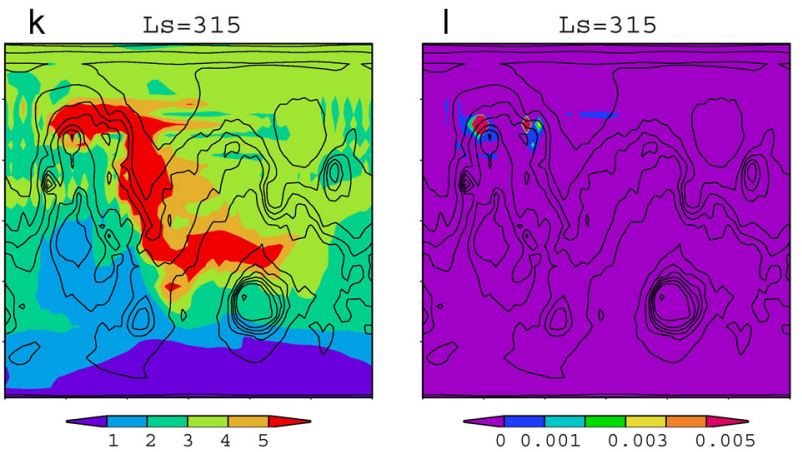

Figure 16. (a, c, e, g, i, k) Geographic distribution of normalized opacity at six time intervals covering the storm evolution. The storm begins $\sim L_{s}=220^{\circ}$ at the northeastern edge of Tharsis. In the first frame, dust is lifted to the north of Alba Patera, and then it spreads to the east in a band that extends into Acidalia. The concentration and enhancement of the Hadley cell southward return flow spread the dust to the eastern side of Tharsis. The storm extends into Chryse and across the equator. It then spreads to the east and north of Hellas. (b, d, f, h, j, l) Net dust lifting per day $\left(\mathrm{gm} / \mathrm{cm}^{2}\right)$ for the corresponding $L_{s}$ is shown in alternate panels. The dust-lifting sites are concentrated in the northern midlatitudes to the north of Tharsis. The topography is contoured in black.

of Tharsis. Within this region, MOC images show the development of frontal dust bands associated with lowpressure frontal storms (cyclones) [Cantor et al., 2001, 2002; Wang et al., 2003]. In the first frame of Figure 16, dust is lifted to the north of Alba Patera. The dust from this source is spread to the east in a band that extends into Acidalia. By the next time step (Figure 16c), the region of very high opacity extends along the western side of the Acidalia-Chryse basins, along the channel of observed frontal storm flushing in 1999 and 2003. This channel is associated with the concentration and enhancement of the Hadley cell southward return flow along the flank of Tharsis, the western boundary current of the AcidaliaChryse lowlands [Joshi et al., 1995; Wang et al., 2003]. By this stage, over $15^{\circ}$ of $L_{s}$ after initiation, dust has spread to fill most of the northern hemisphere with opacities near 2 .
Dust lifting has extended along the edge of the Tharsis plateau from northwest of Alba Patera, along to the east and then to the south, and into Chryse. In later frames, the region of very high opacity is seen to extend across the equator and into the southern hemisphere (Figures 16c, 16e, and $16 \mathrm{~g}$ ). Thereafter, the dust spreads to the east, and to the north of Hellas, in the strong westerlies of the Hadley convergence zone. Throughout the storm, dust lifting remains concentrated in the northern midlatitudes to the north of Tharsis. Dust lifting does not develop secondary centers in the southern hemisphere. However, independent storms in the Hellas region in some simulations rage at the same time as the Chryse storm, both acting as primary lifting centers in their respective hemispheres.

[47] We use the space-time harmonic analysis to separate eastward and westward propagating waves. The eastward 


\section{$P_{s}$ Variance: $\quad$ Lat $=52.5^{\circ}$}
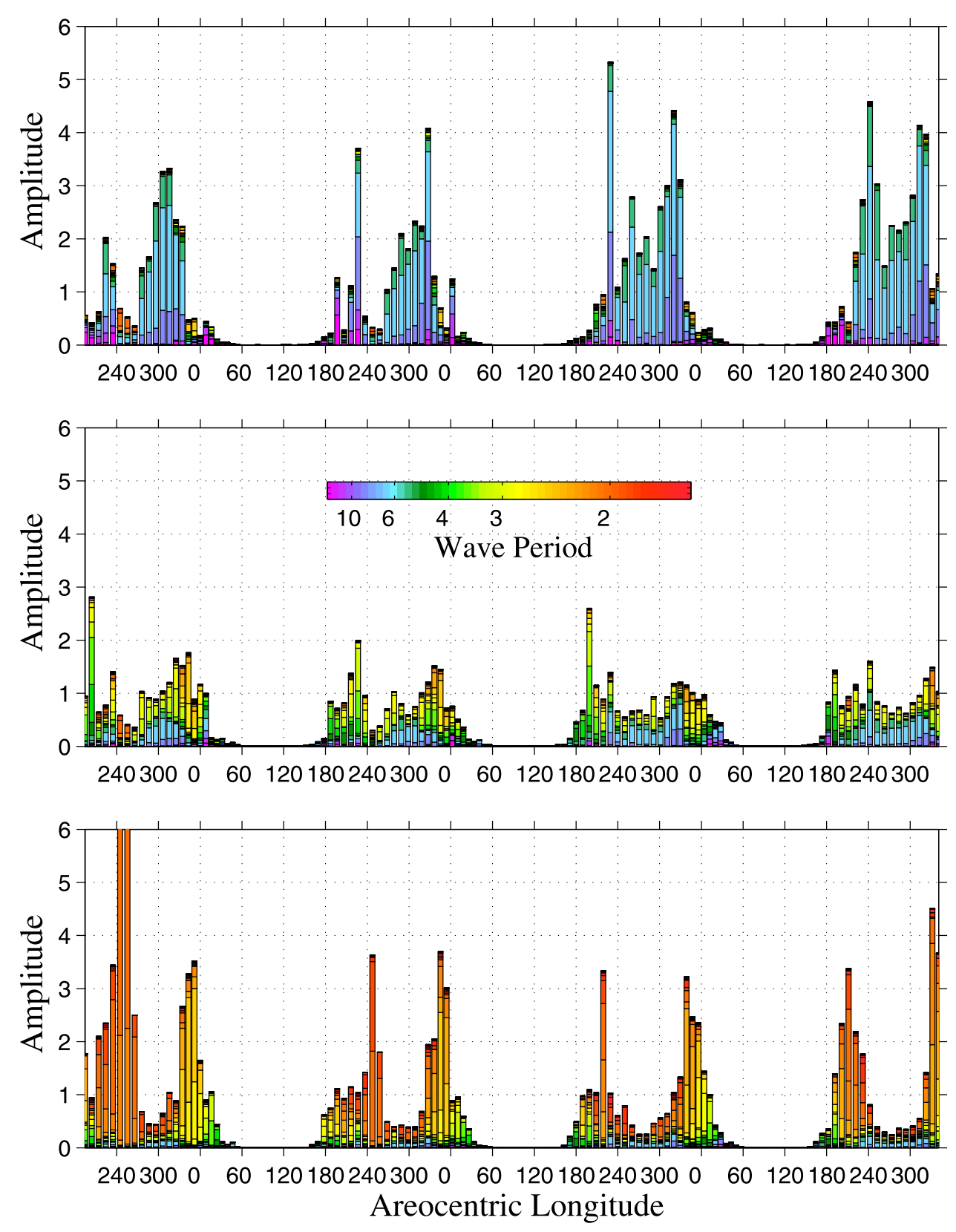

Figure 17. The seasonal variation of eastward propagating zonal waves 1, 2, and 3 (top, middle, and bottom) derived from simulated surface pressure (Figure 3c, year 1). At each $L_{s}$, the contributions to the total variance are represented in color.

propagating waves are found to dominate. This result is consistent with many other studies [Wilson et al., 2002; Banfield et al., 2003; Wang et al., 2005]. The seasonal variation of the eastward propagating zonal waves 1,2 and 3 can be seen in Figure 17. The variable in this case is the surface pressure. At each $L_{s}$, the contributions to the total variance are represented in color. Periods around 6 sols dominate the zonal wave 1 variance (cyan), while the variance of zonal wave number three is dominated by periods of $\sim 2$ sols (red). A clear pattern of presolstice and postsolstice activity is obvious for all the 3 zonal waves. Wave 3 is particularly active in the first year of the simulation. The peak period of wave activity is at $L_{s}=$ $190^{\circ}-240^{\circ}$ and $L_{s}=310^{\circ}-350^{\circ}$. There is very little activity at solstice. Flushing storms are most closely associated with zonal wave 3 since they have comparable seasonal variability. This has also been observed to be true by the analysis of MOC imagery and TES temperature retrievals [Wang et al., 


\section{RMS V (2km)}
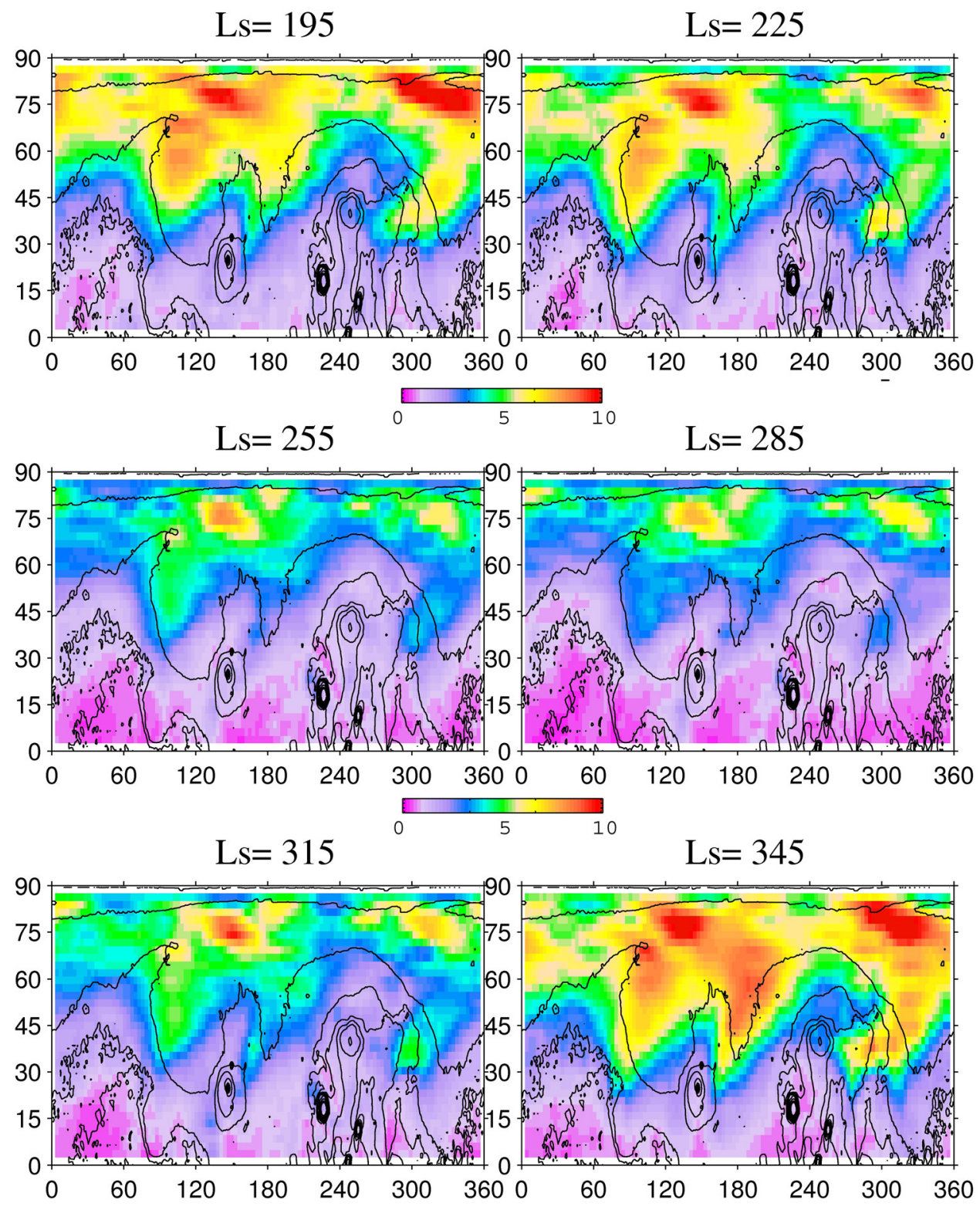

Figure 18. Spatial map of the eddy $\mathrm{V}$ fields at $2 \mathrm{~km}$ above ground for different times of the year (Figure $3 \mathrm{c}$, year 1). The presolstice and postsolstice activity is clear from the plots. Note that the longitude goes from $0^{\circ}$ to $360^{\circ}$ instead of $-180^{\circ}$ to $180^{\circ}$.

2003]. It has also been observed that the zonal wave 3 is associated with a period of $\sim 2$ sols, a result that we obtain in our simulations as well. The TES temperatures indicate that the temperature variance is strongest near the surface; i.e., these traveling waves are shallow, consistent with GCM simulations.

[48] Even though the simulated postsolstice wave activity is not particularly weaker than the presolstice waves, simulated flushing storms are less frequent in the postsolstice period. This is evidently due to the asymmetric southward extension of the $\mathrm{CO}_{2}$ ice cap about the solstice.
In the postsolstice season, regions of highest wind stress tend to be ice-covered, so that dust lifting is not permitted.

[49] Figure 18 shows the spatial maps of the eddy V-fields (at $2 \mathrm{~km}$ ) for different periods in the southern spring and summer season. This can be compared to Wang et al. [2005] and Banfield et al. [2003, Figure 20]. The enhanced activity in the presolstice and postsolstice seasons is clear from these figures. The seasonal variation of eddy temperature and surface pressure fields are comparatively muted by comparison.

[50] The flushing storm events in the MOC imagery analysis are active in all 3 channels: Acidalia (into Chryse); 


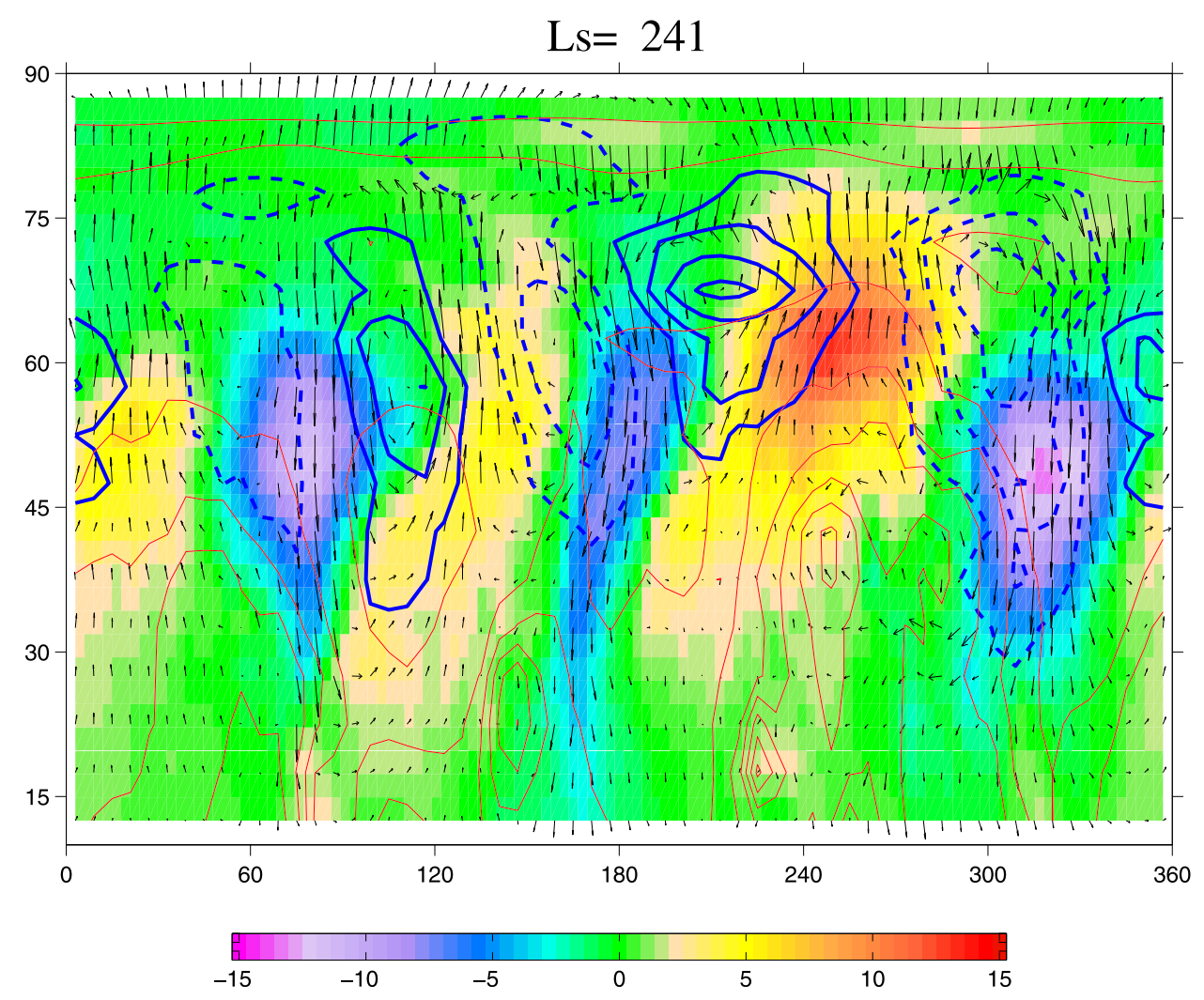

Figure 19. Spatial variation of eddy fields for $L_{s}=241^{\circ}$ (Figure 3c, year 1). The quasi-stationary and tidal waves have been filtered. The shaded field is the eddy temperature field. The eddy pressure field is plotted in dotted (high) and solid (low) contours. The eddy velocity field is shown by the arrows. Note that the longitude goes from $0^{\circ}$ to $360^{\circ}$ rather than $-180^{\circ}$ to $180^{\circ}$.

Arcadia and Utopia [Wang et al., 2003, 2005]. Acidalia is the most active. On the basis of the eddy $\mathrm{V}$ winds in Figure 18, one can see that that storm initiation (and southward propagation) should be favored in all 3 lowland channels (Acidalia, Arcadia and Utopia). The Acidalia/Chryse channel does seem to be the region of most of the flushing storms in our simulations. An examination of simulated stresses shows a prominent diurnal cycle in many places with an additional modulation from traveling waves. In the model, the dust lifting is influenced by both tides and traveling waves. For these cross-equatorial storms, greater dependence on frontal lifting than tides gives greater interannual variability as this captures the transient wave variations. The tides act as gatekeepers to northern weather systems penetrating into the southern hemisphere.

[51] A snapshot of the eddy fields at $L_{s}=241^{\circ}$ is shown in Figure 19. The zonal wave 3 is clearly visible in the eddy temperature, pressure and velocity fields. The fronts are formed at the intersection of the northward and southward eddy winds. At this time, there is a dominant wave 3, 2-sol period traveling wave. There is strong southward motion in the cold regions and northward motion in the warm sectors. The $\mathrm{CO}_{2}$ ice cap has an influence so that the negative temperature anomalies are clamped around $60^{\circ} \mathrm{N}$ latitude because the minimum temperatures cannot fall below the condensation temperature.
[52] The transient waves are stronger in the northern hemisphere compared to the southern hemisphere. They tend to peak around $65^{\circ} \mathrm{N}$. Figure 20 shows the seasonal and latitudinal variation of V-RMS amplitude. The traveling wave activity in the southern hemisphere has a local maximum at $L_{s}=180^{\circ}$. It is likely that these waves combined with the diurnal tide trigger the dust activity in the Hellas basin. The interactions of the retreating $\mathrm{CO}_{2}$ frost cap with the circulation in the Hellas basin possibly introduces some transience that results in occasional storms in the Hellas in the early southern spring season.

\subsubsection{Dust and Air Temperature Distribution for Chryse Type Storms}

[53] The postsolstice temperature cross-section comparisons with TES data for a typical simulated storm year with Chryse storms are shown in Figure 21. The temperatures are in reasonable agreement considering the fact that simulated postsolstice Chryse storms are less frequent than observed postsolstice storms. The TES data are warmer at higher altitudes and near the surface in southern midlatitudes. The gradient at $55^{\circ} \mathrm{N}$ is present in both the data and simulation, but it tilts poleward more strongly with height in the data. The southern polar temperatures are slightly colder in the simulation.

[54] The storm event shown in Figure 22 is intended to mimic a "Chryse" storm. The baroclinically raised dust events intensify moderately as they travel southwards. The 


\section{RMS Amplitude: (1.5-10 days) Zonal Waves 1-3}
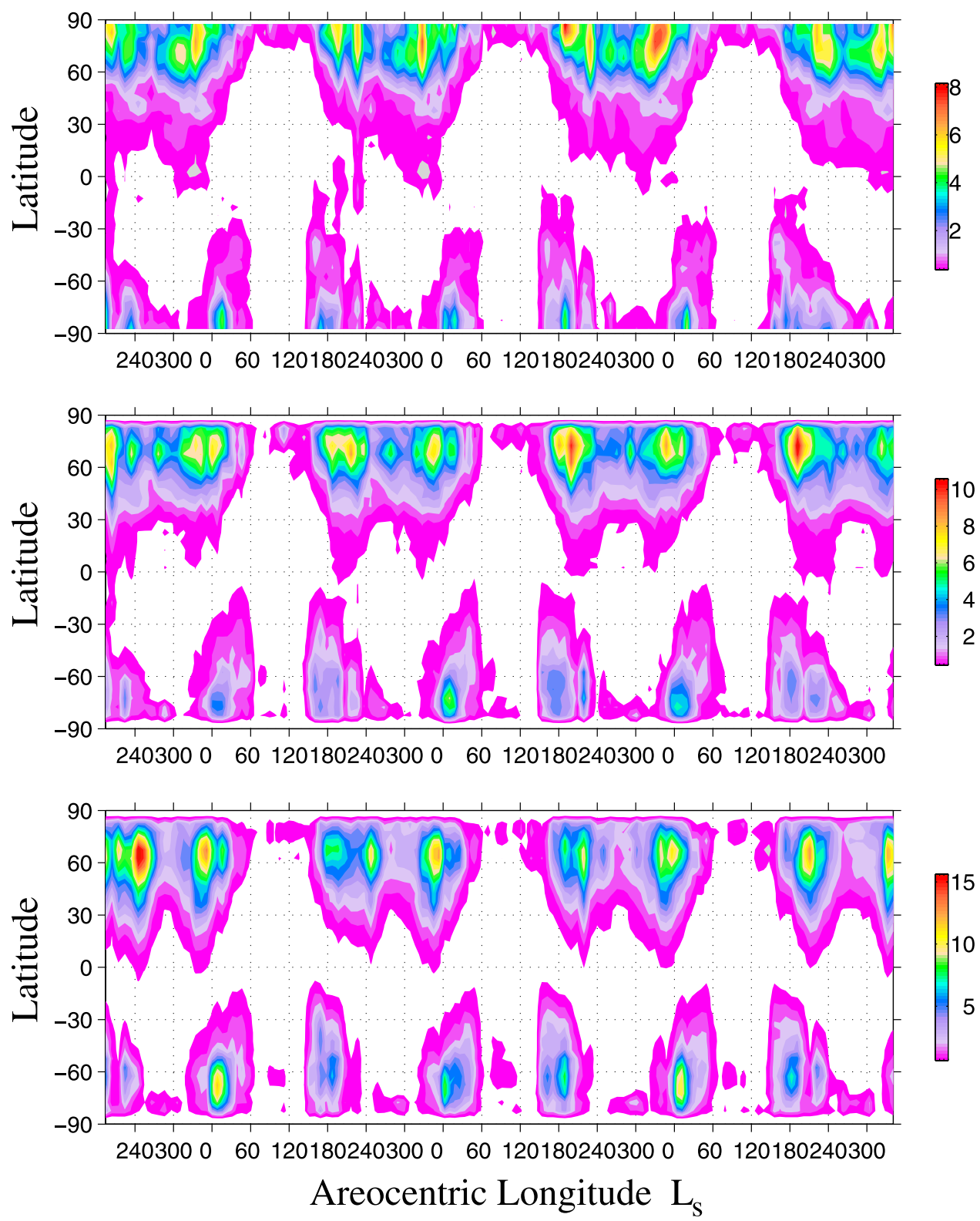

Figure 20. Traveling waves derived from near-surface ( $2 \mathrm{~km}$ above ground) meridional velocity from zonal wave 1 to zonal wave 3 (Figure 3c, year 1). Their periods range from 1.5 days to 10 days, respectively. The amplitude of wave 3 is dominant particularly at latitude $50^{\circ} \mathrm{N}$. A rough variation in latitude can be seen from the plot. This can be correlated with the advance and retreat of the polar caps.

"Chryse event" remains very shallow until dust is exported southward into the Hadley circulation. In this year, an early Hellas storm was missing and hence the Chryse storm can be seen clearly in the cross-section figures.

\section{Global Dust Storm Initiation}

[55] The initiation of the type of storm shown in Figure 22 has been described by Wang et al. [2003]. The initial event which begins the first major dust lifting is associated with a particularly strong baroclinic storm. This initiates dust lifting, which becomes entrained in the Hadley circulation. Wang et al. [2003] demonstrate that favorable baroclinic storm activity for dust lifting occurs in two seasonal "windows" centered upon and separated by the northern winter solstice. A variety of factors influence the strength of these storms and their ability to transport dust from the northern to southern hemispheres, including initial storm center latitude, strength of the high-pressure center behind the low, and most importantly, the timing of storm center propagation through Acidalia [Wang et al., 2003, 2005] with respect to the diurnal tide. The variation of intensity of 

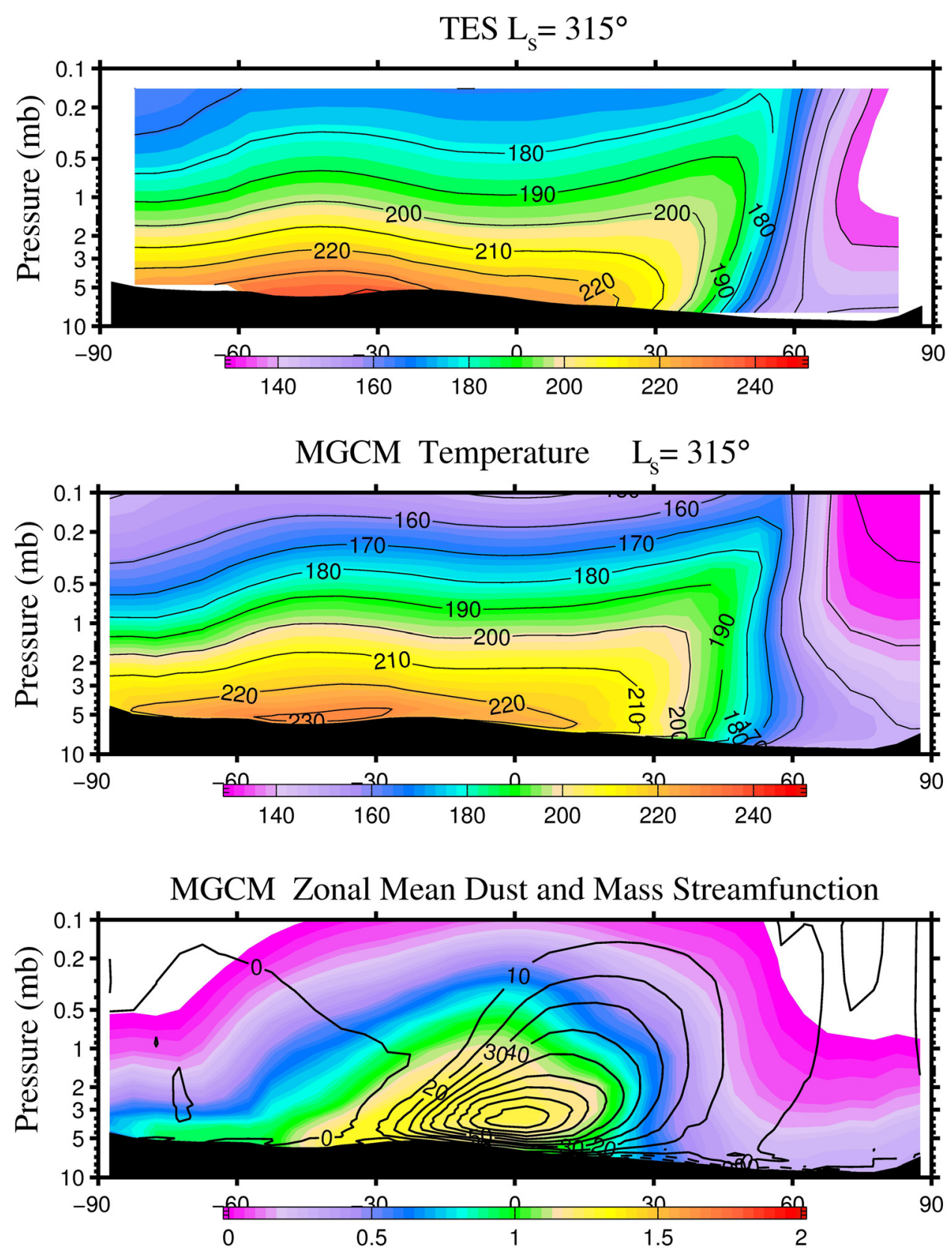

Figure 21. Simulated dust distribution and $T_{15}$ temperatures as a function of latitude and height compared against the MGS TES data for $L_{s}=315^{\circ}$ (Figure 3c, year 2). This is the period when the postsolstice Chryse type storms are most active. The third panel shows the simulated vertical dust distribution (shaded) and mass stream functions $\left(10^{8} \mathrm{~kg} / \mathrm{s}\right)$ (contoured) at this time of the year.

these baroclinic storms provides the "random seed" from which regional or global storms of this type can form. The regional storms from the Hellas basin are also initiated by the transient wave activity arising from the interaction of the retreating south polar caps with the Hellas basin circulation (Figure 12).

[56] The global storms that develop from the Hellas basin do not occur regularly, and it is important to understand what is different in the model in years with and without global storms. The pattern and magnitude of the maximum surface wind stresses just before the initiation of the storm shown in Figure 7, is shown in Figure 23a, and for the same seasonal date in the same simulation for a year without a global storm is shown in Figure 23b. Differencing of the two panels (Figure 23c) shows that just before the initiation of the global storm, the surface winds stresses were roughly $50 \%$ higher on the northern rim of Hellas in the year with the storm. Superposition of transient waves is most likely responsible for higher stresses. It is at this location that the first major dust lifting in the simulated storm begins.

[57] The behavior of wind stresses around the Hellas basin in late southern spring is shown in Figure 24. The year without a global dust storm has predictable stresses that follow the diurnal pattern. However, in the year that has a 

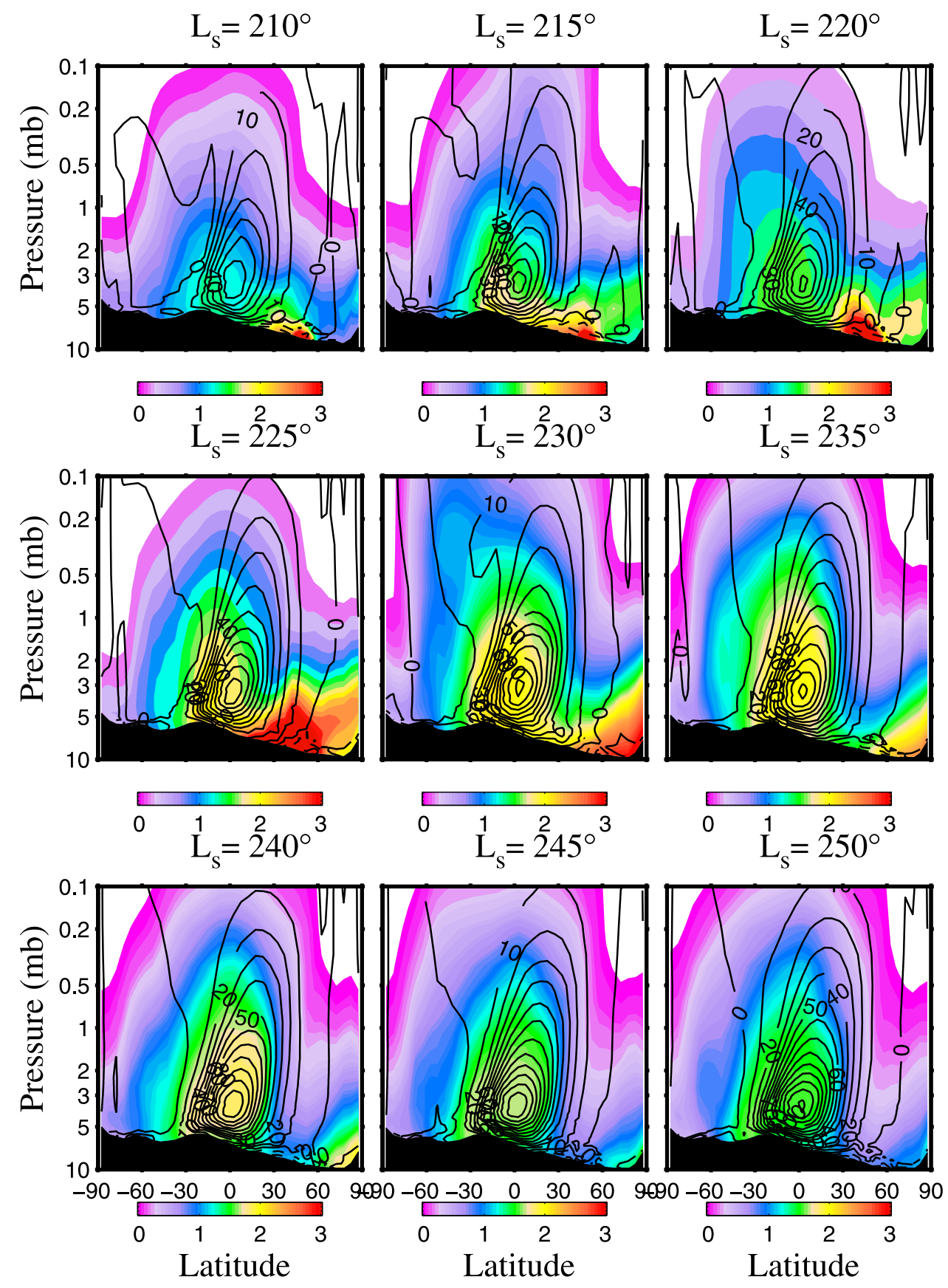

Figure 22. Dust distribution (shaded) resulting from a northern hemisphere dust-lifting event, largely in the Chryse region (Figure 3c, year 1). The zonal mean tropical temperature at $L_{s}=240^{\circ}$ is comparable to the temperatures in this season in subsequent years though the time evolution is different. A critical difference appears to be the lack of an early-season $\left(L_{s}=205^{\circ}\right)$ storm event in Hellas that we see in some of our other simulations (Figure 3c, year 2). The mass stream functions $\left(10^{8} \mathrm{~kg} / \mathrm{s}\right)$ are contoured in black.

global storm, the stress pattern has additional transience that gives rise to high values of stresses that exceed the stress threshold. The mean and variance (interannual) of stress evaluated over 10 years in the period between $L_{s}=230^{\circ}$ and $240^{\circ}$ are shown in Figure $25 \mathrm{a}$, with the maximum wind stresses at each point in the periods sampled shown in Figure 25b. The basin itself is associated with low stresses and weak variability. The high stresses are associated with flows up and down the rim of the basin. These are diurnally varying slope winds that peak in the afternoon. The peak mean stresses are found on the northwestern rim of the basin, with the maximum variance extending from the west and south, along the western and northern rim. The northwestern rim is along the same latitude as the subtropical jet, which helps in the attainment of elevated stresses in this region. The maximum variance is found to the south west of the basin. This variance arises from traveling wave activity that peaks near the south polar seasonal cap around $L_{s}=$ $180^{\circ}$. For this reason the southwest corner of the Hellas basin is an active lifting center earlier in the season and not 

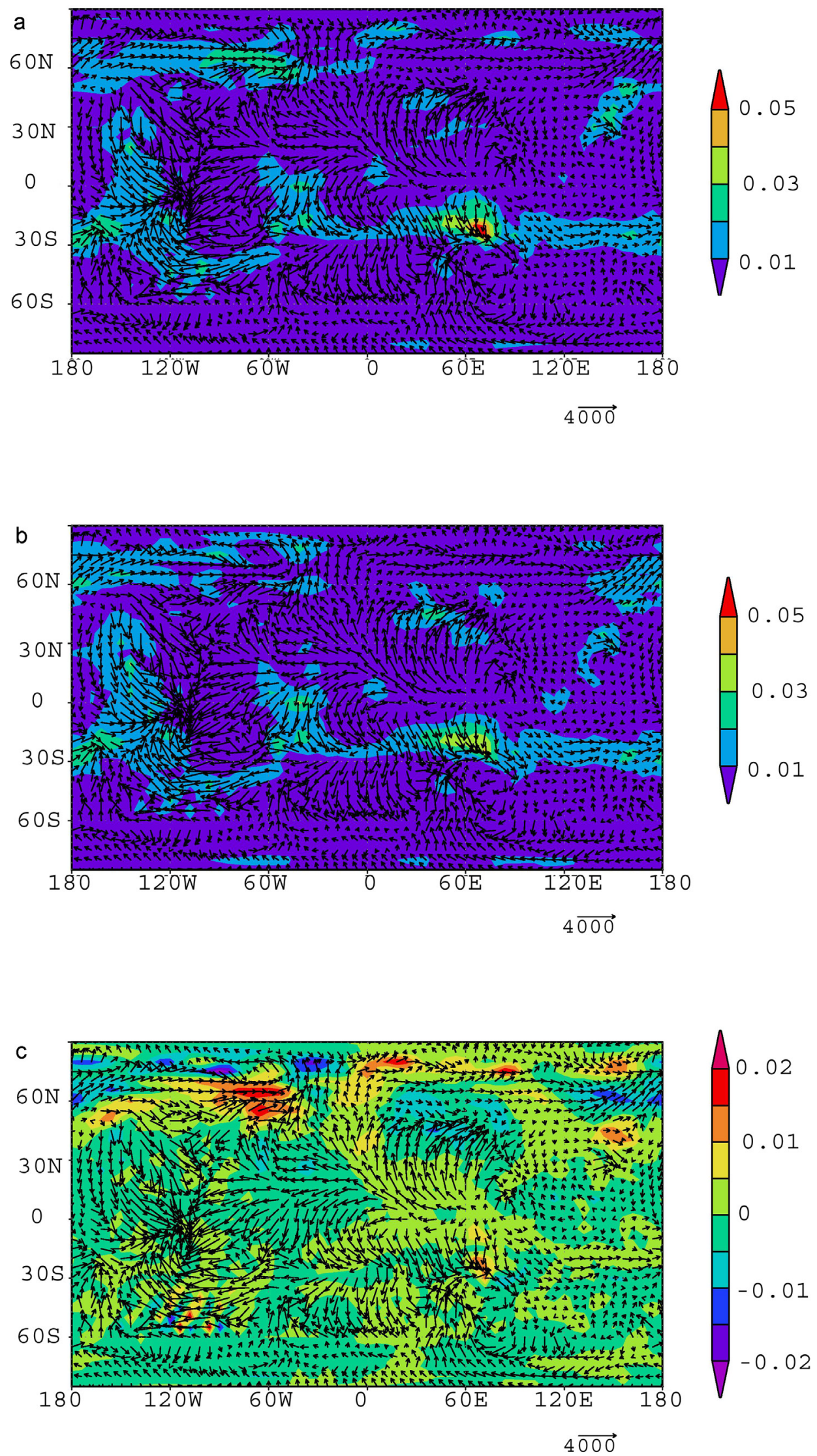

Figure 23. Spatial map of stresses (Pa) and wind (a) just before the beginning of a global storm (Figure 3b, year 3) and (b) for a year with no global storm (Figure 3b, year 2). (c) The difference in stresses. 

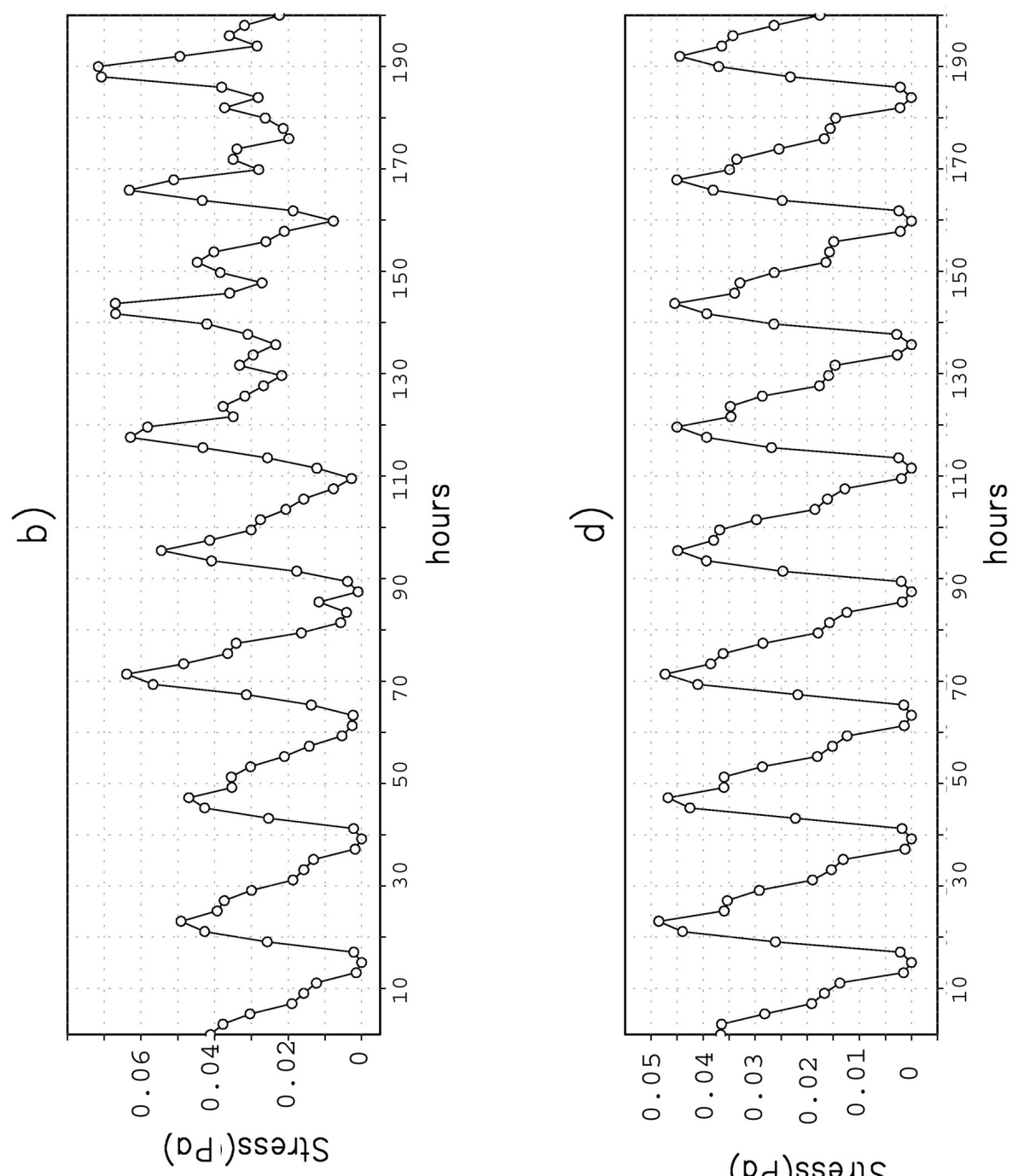

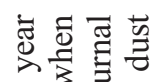

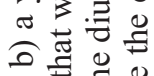

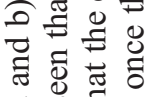

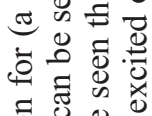

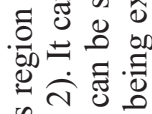

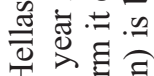
के की . 的远导 ปี

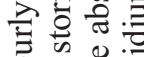
实商焉 छ

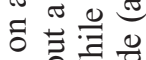
졸

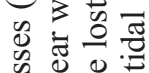
牙

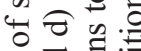

을 蒠总 (Dd)ssarts
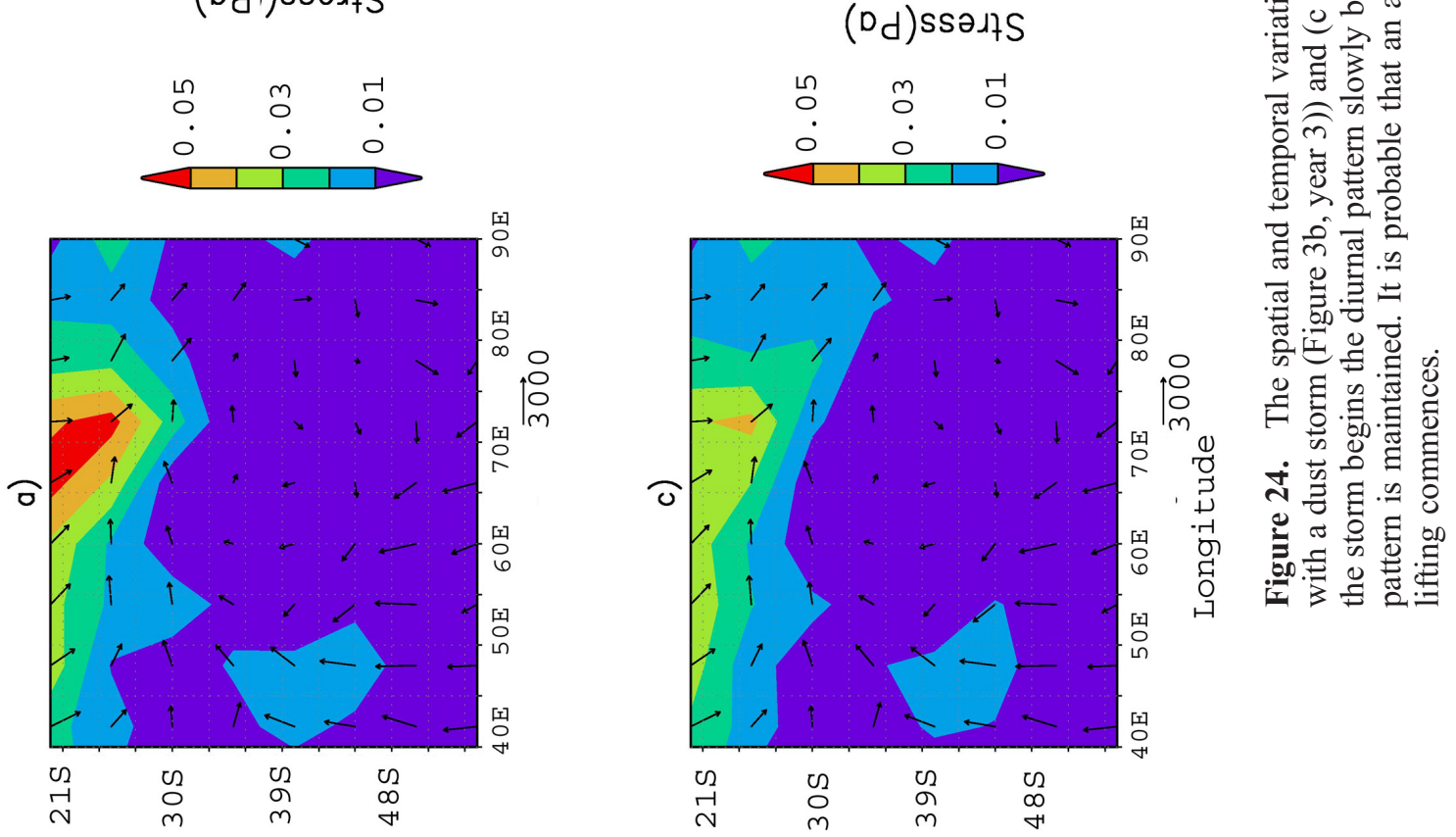
so active later when the subtropical jet is more important for triggering storms from the northern rim of Hellas. These patterns of maximum variance in the stress are consistent with the locations of dust lifting prior to the development of the 2001 dust storm. The maximum wind stresses also echo this pattern, with peak values on the highlands just north of the basin, to the south and west of the basin, and along the western rim. Peak prestorm stresses exceed 0.045 Pa. As the circulation within and near the Hellas basin is driven largely by the diurnal variation of heating and associated slope flow into and out of the basin, the day-to-day variance is associated with slight differences in the strength of these diurnal flows. The precise dynamical origin of these differences is less obvious than for the variability of stress in Acidalia, and further study is deferred to a later paper. What is clear, however, is that "weather noise" (most likely introduced by the interaction (reinforcement and cancellation) of traveling waves/various tidal components whose phasing is sensitive to the background dust distribution, as well as topography [Zurek and Leovy, 1981]) associated with the Hellas basin flow allows stresses in rare occasions to exceed the threshold for dust lifting and to such a degree that a global dust storm is sparked. The traveling waves resulting from the retreating south polar cap thermal circulation might also be instrumental in providing this weather noise for storm triggering in the Hellas basin (for the earlyseason storms). The traveling waves are weaker in the southern hemisphere compared to the northern hemisphere, but observations indicate that they have an important role to play in the interannual variability of the global dust storms arising out of the Hellas and Argyre basins [Wang et al., 2003].

[58] Even though simulated regional storms can be initiated as early as $L_{s}=185^{\circ}$, the global storms do not start that early in the season. In the absence of mesoscale circulations in our model (e.g., Solis Planum and Daedalia which acted as secondary lifting centers for the 2001 GDS), the early Hellas storms are not able to sustain themselves without any help from these additional lifting centers.

\section{Dust Storm "Switch-Off" Problem}

[59] The comparison between observed and simulated global dust storms shows one substantial discrepancy. While the simulated storms initiate at a reasonable range of seasonal dates and develop peak air temperatures consistent with the observations, the storms do not terminate as observed. The Hellas storms early in the season (Figure 3c) are much smaller. These are helped by the westerlies and the storm spreads in the southern hemisphere due to advection of dust. As the midlatitude westerlies weaken with the approaching summer solstice season along with weakening of traveling waves and melting of polar ice caps from the rims of Hellas, these storms decay. However, the storms that initiate around southern summer solstice in the simulation shown in Figure $3 \mathrm{~b}$ illustrate the problem. The growth rate and peak temperatures of the storms are consistent with observed behavior. However, observations, although limited, would suggest that after about $L_{S}=260^{\circ}$, when the storms have reached their initial peak, decay ought to set in. For example, both the $1977 \mathrm{~b}$ and 2001 storms began to decay about $20^{\circ}-30^{\circ}$ of $L_{s}$ after initiation [Martin and
Richardson, 1993; Smith et al., 2002] and that there was no "rounding off" of storm activity at a sustained peak; explosive growth was immediately followed by rapid decay. By contrast, the simulated $L_{s}=230^{\circ}$ storm remains active and in a fully developed state from before $L_{s}=260^{\circ}$ until just before $L_{s}=315^{\circ}$. Only after $L_{s}=315^{\circ}$ does the storm decay. Once it does start to decay, however, it does so at a rate that is similar to that of observed storms, which is essentially controlled by the dust particle sedimentation rate since there is cessation of lifting. Thus, after the termination of dust lifting in the storm, the model emulates the observations well, which is consistent with previous storm-decay experiments with GCMs [e.g., Murphy et al., 1995; Wilson and Richardson, 1999]. The problem, then, is not the decay process itself, but "triggering" of the decay phase. Simply put, the dust source does not quickly shut down in our simulations.

[60] The feedback between increased atmospheric dust opacity, increased circulation vigor, and increased dust lifting is an essential part of explosive GDS development, and is well captured by the GCM for the given resolution of the model. However, it is apparent that at some point either this feedback must become inactive, or the dust in the source region must deplete. In these simulations we have assumed an infinite supply of dust and hence the dust is never depleted from the lifting centers. It has variously been speculated that the development of strong static stability in the boundary layer associated with high opacity causes a reduction in wind stresses and a cessation of dust lifting [Newman et al., 2002a]. This negative feedback mechanism might be instrumental in storm decay on Mars. This feedback issue has also been discussed by Haberle et al. [1993] and Murphy et al. [1995].

[61] Although the GCM does develop an increasingly stable boundary layer at storm peak, it does not reduce stresses dramatically and does not cut off dust lifting. This is possibly due to the coarseness of the vertical grid that fails to capture small signatures of critical processes and the lack of the full dust and circulation feedbacks in the model.

[62] The GCM GDS decay sets in by the spin-down of the Hadley circulation in late southern summer. As the Hadley circulation naturally slows as the subsolar point returns equatorward, stresses in the Hadley cell convergence zone (subtropical jet) (which predominates GDS dust lifting) decrease to a point where lifting stops. There is a feedback here (Figure 2). As the Hadley cell weakens due to changes in solar forcing, the amount of dust lifting and hence opacities are reduced. This causes a further weakening of the Hadley circulation. Eventually, active stress lifting terminates and the GCM drifts back to a low dust state. The results of the low-order model of Pankine and Ingersoll [2002] can also be interpreted in this manner. In that case, the model was limited in the range of possible behavior. However, the generation of similar behavior in a full circulation model suggests that storm switch-off may be a significant challenge if internal feedbacks are of dominant importance.

[63] Another option for storm switch-off is source region depletion. There is reason to expect that the dust deposits in the source regions are not uniformly more than a few millimeters to a few centimeters deep (otherwise they would appear as "dust" in visible and thermal infrared retrievals, 

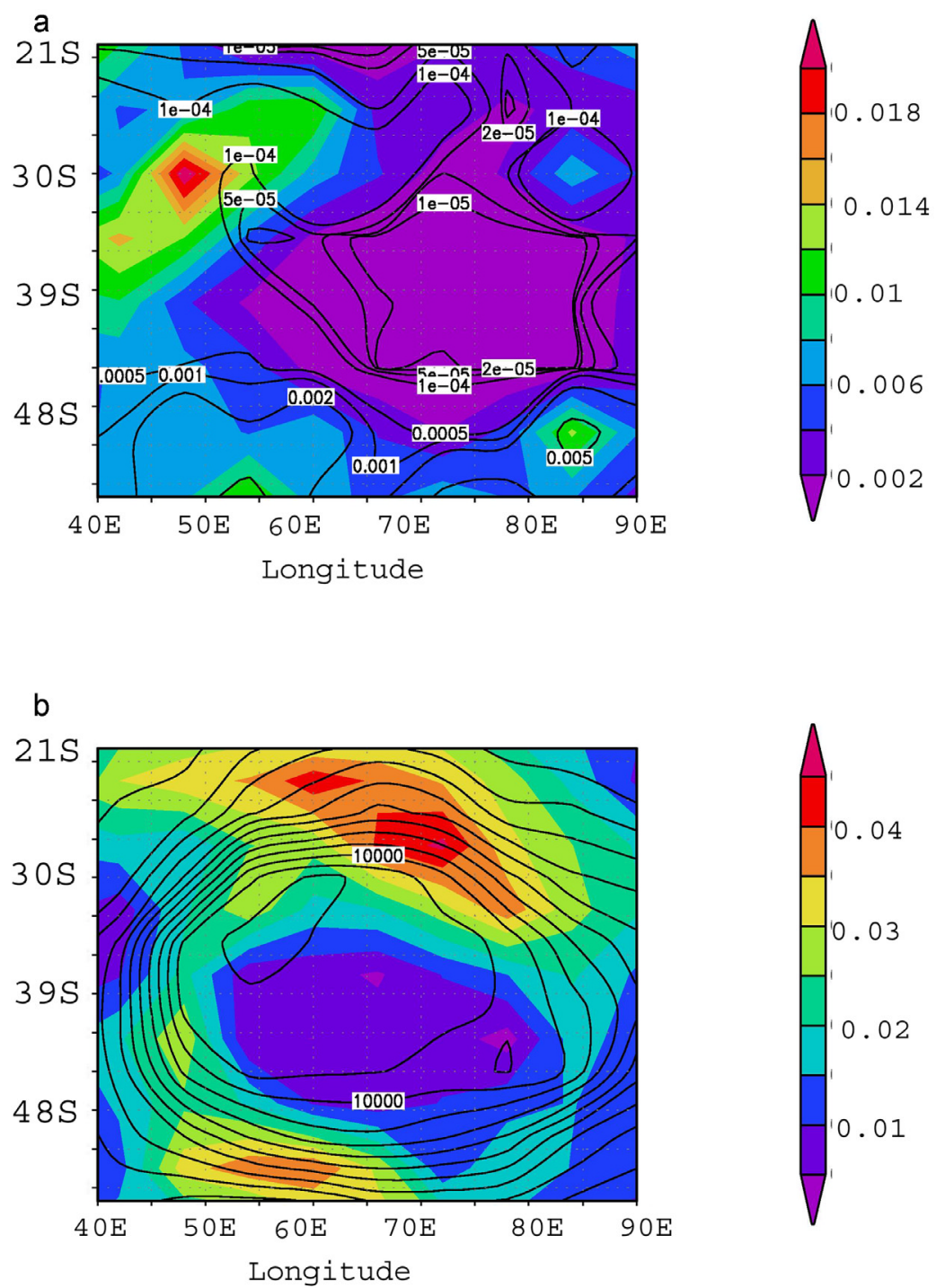

Figure 25. (a) The mean (shaded) and variance (contours) of stress (Pa) evaluated over 10 years in the period between $L_{s}=230^{\circ}$ and $240^{\circ}$ (Figure $3 \mathrm{~b}$ simulation). (b) Spatial distribution of maximum wind stresses at each point in the periods sampled.

which has a low thermal inertia signal). During a large, sustained dust storm, the GCM predicts dust removal in source regions of about this thickness, while the predicted fallout would take over 10 years to resupply this much dust. In addition to dust fall out, dust devils and other regionalscale circulation components also supply dust to expired sites. Exhaustion of dust source regions during a dust storm would thus seem plausible (S. Basu et al., manuscript in preparation, 2006).

[64] Some initial simulations have been undertaken with the GCM using exhaustible surface dust deposits. As might be expected, the storms develop sharp temperature and dust opacity maxima as the primary source region depletes. The trend of air temperature in these simulations mimics that of the real atmosphere much better than that of the "infinite dust source" model, shown in Figure 3. These simulations provide a much richer variety of behavior as the surface distribution of dust becomes an additional source of internal model variability. Discussion of simulations with exhaust- able surface dust deposits is deferred to a later paper. Simulations with larger particle sizes also gave faster rates of storm decay as the sedimentaion rates are higher in this case.

[65] Sensitivity to heating rates by changing the single scattering albedo has also been tested. Even though the start times for some of the storms was a little earlier than the default case, there was no effect on the storm decay.

\section{Net Dust Transport by Global Dust Storms}

[66] The net annual dust deposition/erosion predicted by the GCM for non dust storm years has been shown and discussed by Basu et al. [2004]. For completeness, and in order to illustrate the residual signature of a GDS in the annual surface dust deposits, the annually integrated dust deposition/erosion for years without and with a GDS are shown in Figure 26. The non dust storm year shows dust erosion on the southwestern rim of Hellas, in Acidalia/ 

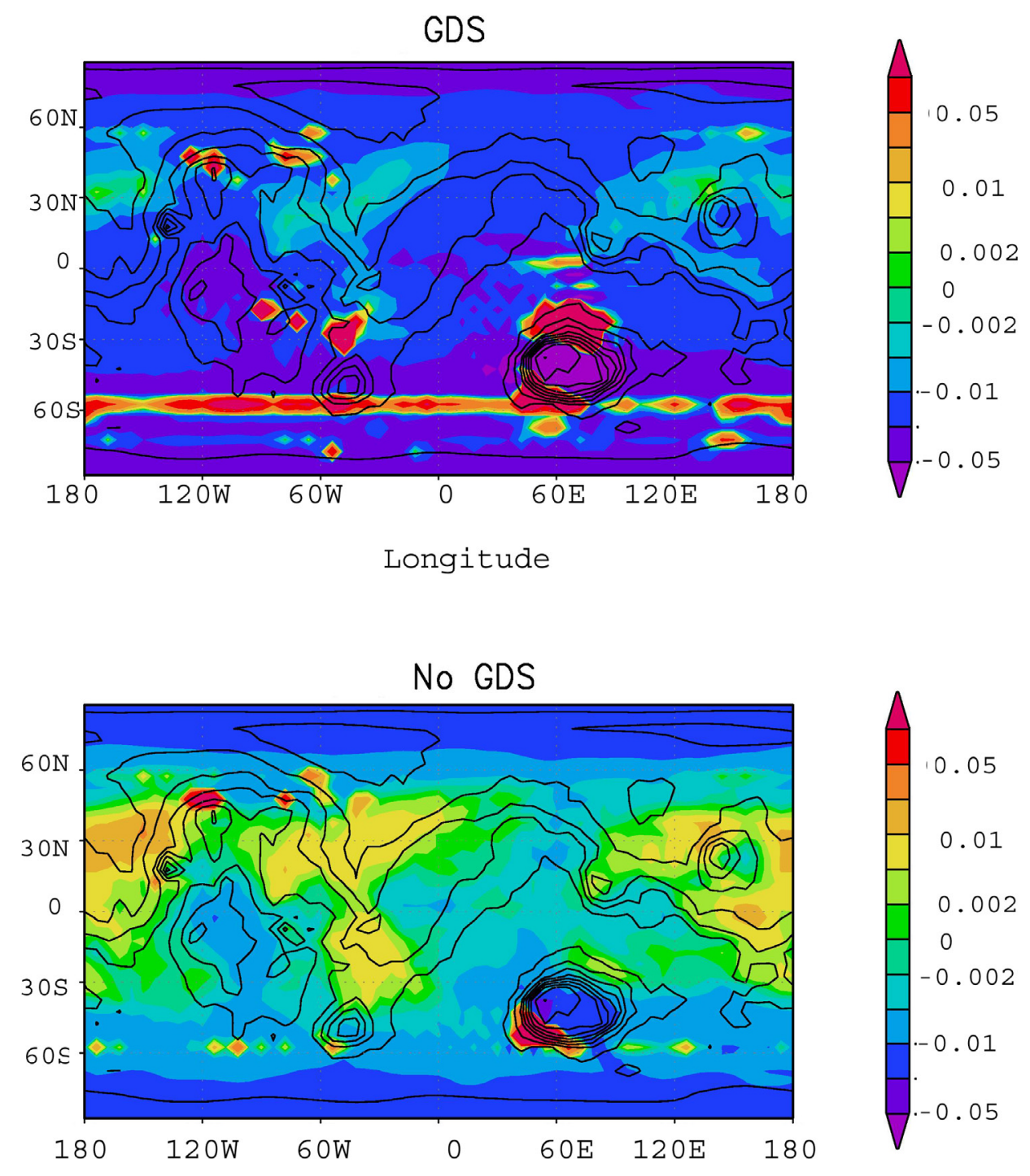

Figure 26. The net annual erosion $\left(\mathrm{gm} / \mathrm{cm}^{2}\right)$ predicted by the GCM for years with (Figure $3 b$, year 3) and without a global dust storm (Figure 3b, year 2). The topography is contoured in black. The effect of the GDS is to generate large erosional signals in the storm source regions: northern and southwestern rims of Hellas and points within Syria and Solis and north of Argyre. The non dust storm year shows erosion on the southwestern rim of Hellas, in Acidalia/Chryse, Amazonis and northern plains to the west and south of Elysium. High deposition rates occur at both poles, on Tharsis, and in the Hellas basin. Increased deposition from dust fallout in the GDS case results in broad areas of net sinks that were net sources in the no GDS case.

Chryse $\left(45^{\circ} \mathrm{N}-45^{\circ} \mathrm{S}, 30^{\circ}-60^{\circ} \mathrm{W}\right)$, in Amazonis, and on the northern plains to the west and south of Elysium. High deposition rates occur at both poles, on Tharsis, and in the Hellas basin. This simulation is discussed in more detail by Basu et al. [2004].

[67] The effect of the GDS is to generate large erosional signals in the storm source regions. In this case, those regions are the northern and southwestern rims of Hellas and points within Syria and Solis, and to the north of Argyre. These regions were observed to be active during the 2001 GDS [Strausberg et al., 2005]. The GDS has a major effect on the surface deposits at most other locations. Increased deposition resulting from dust fallout in the wake of the GDS results in broad areas that were net dust sources becoming net sinks. In fact, the majority of the tropics and lower midlatitudes are predicted to be net annual sinks during a year with a GDS, as might be expected.

\section{Discussion and Summary: Interannual Variability of Global Dust Storms at This and Other Climatic States}

[68] Mars currently exhibits interannual variability of GDS development. On the basis of the results shown by Basu et al. [2004], there is a very limited range of injection scheme parameters that allow variable GDS development in 
the GCM. In particular, the stress-lifting threshold must be within a range only $10-20 \%$ (of the optimal stress threshold) wide. The fact that Martian dust storms are variable immediately suggests that initiation is a "threshold" problem: one gets a storm when a certain threshold is exceeded, and the system is close to that threshold. Realization that surface wind speeds and stress likely change dramatically with orbital parameters [Fenton and Richardson, 2001; Haberle et al., 2003] presents an obvious question: are we observing Mars at a special time in its history, a time when GDS events are variable? At higher obliquity do GDSs occur regularly, while at low obliquity they never occur? Or does the system adjust so as to keep GDSs variable? The extremely low probability of observing Mars during a "special time" makes the former possibility unpalatable.

[69] Maintenance of interannual variability requires a negative feedback mechanism which returns the system to a state of variable GDS development; it must get harder to generate a GDS as more GDSs develop, and easier as fewer develop. Ultimately, this mechanism must involve the surface dust deposits themselves and the ease with which the atmosphere can lift dust from them. It is possible to speculate about the nature of such mechanisms. Pankine and Ingersoll [2002, 2004] suggested that the local distribution of dust on the scale of rocks and individual "roughness elements" might be important. One can imagine a situation, such that when winds increase in strength during periods of higher obliquity, dust is only trapped on the surface in deposits that are shadowed from the wind (in rock crevices or trenches). Higher stresses would then be needed to generate a given dust storm event. Conversely, as surface winds decrease, the dust could be removed from shadowed regions by dust devil or other convective processes until the dust is located in sufficiently exposed locations that the wind stresses can again lift it. In this situation, a lack of GDS activity allows dust devil action to dominate, and spread the dust uniformly, while the development of excessive GDS activity would tend to "hide" the dust. The use of a finite surface dust supply would introduce a new set of initial conditions, in the form of available surface dust, being present at the beginning of each storm season. This is likely to increase the interannual variability in the system.

[70] On the other hand, the feedback mechanism may have nothing to do with the local-scale dust distributions. Instead, the regional-scale distribution of dust may be critical. Figure 1 shows the highest wind stresses experienced on the planet in a given annual cycle for a year without a GDS. There is strong spatial heterogeneity apparent in the map, and while the details of this distribution may change somewhat as obliquity changes (though not much [Fenton and Richardson, 2001; Basu et al., 2004, Newman et al., 2005; Haberle et al., 2003]), strong heterogeneity is always present. A feedback mechanism involving the geographical distribution of dust then comes to mind. The drive for GDS activity becomes stronger as obliquity increases due to intensified Hadley cell circulation and hence stronger subtropical jet and stronger surface stresses. As this happens, dust is removed from the most easily eroded locations (such as the southwestern and northern rims of Hellas, Syria/Solis, and the northern storm tracks). As the prime sites are eroded, GDSs can only develop if stresses become sufficient to activate lifting in regions where storms could not previously initiate. As the winds and stresses increase with obliquity, the dust would "roll back" (be cleaned out) progressively from the most easily eroded regions and would remain only in the geographical locations that exhibit lower wind stresses. This process would continue until a steady state is reached with variable GDS activity. The steady state is maintained when a balance is struck, for a given region, between the multiannual-average export of dust by GDSs and import due to fallout of dust lofted by dust devils elsewhere on the planet. In this way, GDS activity would be maintained in an interannually variable mode until the planet was uniformly covered by dust at the low-obliquity extreme or dust was entirely removed from the exposed surface and locked up in ice sheets (in the tropics! [Mischna et al., 2003]) at the high-obliquity extreme. In fact, the low-obliquity extreme may be limited by the deflation of the atmosphere after the formation of permanent $\mathrm{CO}_{2}$ ice caps.

[71] The tendency for a climate model to lock itself into a variable GDS regime when a negative feedback system is imposed has been illustrated in a low-order model [Pankine and Ingersoll, 2004]. Indeed, once one decides to put such a feedback system into such a model, the outcome is unavoidable. Experiments with a GCM with active and exhaustible dust deposits are required to further explore the likelihood that Mars is driven to find such a steady state. The GCM will have the freedom to internally generate a spatial dust source feedback system (i.e., as an emergent behavior, not externally imposed on the model) and as such, the model possesses the potential to surprise. If the feedback involves the microscale distribution of dust, however, parameterized mechanisms may still need to be imposed.

\section{Summary}

[72] This paper reports on the successful generation of global dust storms within a GCM that simultaneously provides a good simulation of the non dust storm climate [Basu et al., 2004]. The dust storms develop spontaneously within the GCM, without ad hoc forcing, during southern spring and summer, consistent with observations. Simulated global storms are seeded by spontaneously generated local events, and grow by a radiative-dynamical feedback involving increased radiative heating associated with the lofted dust, increased Hadley cell vigor, and increased wind stresses and dust lifting. Growth from regional to global scales involves the activation of secondary lifting centers. During the simulated global storms, dust is lifting from a relatively small number of sites where wind stresses are maximized. Common areas are the rims of the Hellas and Argyre basins, the southern seasonal cap edge and Acidalia.

[73] In our simulations, model-resolved wind stresses are responsible for the generation of simulated storms by the dust-lifting parameterization. Only when the threshold stress for lifting is set low enough, and the injection rate parameter (linking the stress function to the injection rate) is set high enough, do global storms develop. The GCM develops interannually variable global storms in a limited area of this phase-space. Within a given continuous simulation, the model will generate years without any major storm activity, interspersed with years with global storms of 
various size and specific timing of initiation. This variability is internal to the atmosphere and the $\mathrm{CO}_{2}$ cycle, as the surface dust deposits do not deplete, and the surface albedo and thermal inertia are not modified by the presence of dust. It is possible that additional richness in the complexity of the dust cycle will emerge in a model with an interactive surface dust distribution, but this work suggests that interannually variable storms are an emergent property of the smoothly and periodically (annually and seasonally) forced system. The interplay with a surface memory site might be an additional source of variability.

[74] Regional and global dust storms have been observed in the Hellas basin, Argyre, Noachis, Amazonis, Hellespontus, Syrtis, Thaumasia, Solis, Claritas, Meridiani and Chryse regions. These big storms start in the southern hemisphere spring/summer season. A truly realistic Martian dust cycle would capture the various dust-lifting centers, have interannual variability in these storms in terms of the intensity of the storm, beginning season and the place of initiation and have a realistic background temperature cycle matching the observations. Our simulations have been able to capture most of these aspects of the Martian dust cycle. However, the simplistic lifting parametrization and spatial resolution in our model does not capture the local circulation and threshold conditions at some the locations like Solis, Noachis and Hellespontus. Hence these are not active lifting centers in our simulations. Most of our storms start in the Hellas and Argyre basins as these have the highest stresses. The dust lifting at other regions is overwhelmed by Hellas and Argyre, which play a major role in initiating and maintaining the global dust storms. When these regions are artificially shut off, and a lower stress threshold is set, the regional storms start off from other lifting centers some of which are in the northern hemisphere like Tharsis and Chryse, however, the feedback necessary for a GDS is not sufficiently invoked. There are not enough data available to implement a variable wind stress threshold map for Mars. But looking at observations it is almost certain that stress threshold varies from one region to another depending on the ease of dust lifting from the surface which in turn depends on surface roughness, local circulations, interparticle cohesion and other such factors.

[75] The simulated storms remain unrealistic in some ways. The most significant being the inability of the storms to shut down in the observed manner. For example, the 2001 global storm began its decay phase around $L_{S}=214^{\circ}$ [Smith et al., 2002], about $35^{\circ}$ of $L_{s}$ after initiation. However, the simulated storms do not decay until the Hadley cell begins to spin-down in mid-southern summer. Storm switch-off must result from either an internal feedback mechanism that kicks in once opacities reach a specific level, or from depletion of the active surface dust supply. The physical processes that would have to be involved in the former are inherently included in the model, but do not generate such a feedback. While it is possible that the fidelity of their representation needs to be improved, the model shows no tendency toward spin-down at high opacity, but the reverse. The latter mechanism is not included in the standard model, initial work with exhaustible sources suggest (not surprisingly) that realistic storm evolution, including switch-off can be simulated. More work is clearly warranted on storm spin-down. In order for there to be two global storms in a year, the first must happen in early-to-mid southern spring. Given that the model is quite capable of generating early- and late-season regional storms, it seems likely that once the spin-down or switch-off problem is solved, dual global storm years will emerge. The preliminary simulations with higher resolution $\left(2^{\circ} \times 2.4^{\circ}\right)$ show promising results. These have storms that start as early as $L_{s}=180^{\circ}$ from the Hellas basin followed by a second storm after the first one decays. The simulated $T_{15}$ temperatures for these storms, however, are lower than observed.

[76] Finally, the range of locations on the surface at which storms initiate and at which secondary dust-lifting centers are generated is in pleasing agreement with observations (the western and northern rim of Hellas, Syria in the southern part of Tharsis, and in the northern midlatitudes in the Acidalia/Chryse "flushing storms" channel [Wang et al., 2003]), the range of behavior seems a little restricted compared to observations. After a while, one recognizes similar types of storms being generated, albeit in a continuously randomized sequence.

[77] Additional variability may require interaction with depletable surface dust deposits and one in which the surface albedo and thermal properties depend on the amount of dust present. Such feedback with the surface may also be necessary to explain the occurrence of variable global storms, despite the fact that a very limited area of model domain space permits these kinds of storms. We speculate, as do Pankine and Ingersoll [2004], that a negative feedback system may pull the Martian climate toward a state with variable storms.

\section{References}

Banfield, D., B. J. Conrath, M. D. Smith, P. R. Christensen, and R. J. Wilson (2003), Forced waves in the Martian atmosphere from MGS TES nadir data, Icarus, 161, 319-345.

Basu, S., M. I. Richardson, and R. J. Wilson (2004), Simulation of the Martian dust cycle with the GFDL Mars GCM, J. Geophys. Res., 109, E11006, doi:10.1029/2004JE002243.

Briggs, G. A., W. A. Baum, and J. Barnes (1979), Viking Orbiter imaging observations of dust in the Martian atmosphere, J. Geophys. Res., 84, $2795-2820$.

Cantor, B. A., P. B. James, M. Caplinger, and M. J. Wolff (2001), Martian dust storms: 1999 Mars Orbiter Camera observations, J. Geophys. Res., 106(E10), 23,653-23,687.

Cantor, B., M. Malin, and K. S. Edgett (2002), Multiyear Mars Orbiter Camera (MOC) observations of repeated Martian weather phenomena during the northern summer season, J. Geophys. Res., 107(E3), 5014, doi:10.1029/2001JE001588.

Clancy, R. T., S. W. Lee, G. R. Gladstone, W. W. McMillan, and T. Rousch (1995), A new model for Mars atmospheric dust based upon analysis of ultraviolet through infrared observations from Mariner 9, Viking, and Phobos, J. Geophys. Res., 100(E3), 5251-5264.

Fenton, L. K., and M. I. Richardson (2001), Martian surface winds: Insensitivity to orbital changes and implications for aeolian processes, J. Geophys. Res., 106, 32,885-32,902.

Fenton, L. K., J. C. Pearl, and T. Z. Martin (1997), Mapping Mariner 9 dust opacities, Icarus, 130, 115-124.

Forget, F., F. Hourdin, R. Fournier, C. Hourdin, O. Talagrand, M. Collins, S. R. Lewis, P. L. Read, and J.-P. Huot (1999), Improved general circulation models of the Martian atmosphere from the surface to above $80 \mathrm{~km}$, J. Geophys. Res., 104, 24,155-24,175.

Gierasch, P. J. (1974), Martian dust storms, Rev. Geophys., 12, 730-734. Greeley, R., N. Lancaster, S. Lee, and P. Thomas (1992), Martian eolian processes, sediments, and features, in Mars, edited by H. Kieffer et al., pp. 730-776, Univ. of Ariz. Press, Tucson.

Greeley, R., M. R. Balme, J. D. Iversen, S. Metzger, R. Mickelson, J. Phoreman, and B. White (2003), Martian dust devils: Laboratory simulations of particle threshold, J. Geophys. Res., 108(E5), 5041, doi:10.1029/2002JE001987. 
Haberle, R. M. (1986), Interannual variability of global dust storms on Mars, Science, 234, 459-461.

Haberle, R. M., C. B. Leovy, and J. B. Pollack (1982), Some effects of global dust storms on the atmospheric circulation of Mars, Icarus, 50, $322-367$.

Haberle, R. M., J. B. Pollack, J. R. Barnes, R. W. Zurek, C. B. Leovy, J. R. Murphy, H. Lee, and J. Schaeffer (1993), Mars atmospheric dynamics as simulated by the NASA Ames General Circulation Model: 1. The zonal mean circulation, J. Geophys. Res., 98, 3093-3123.

Haberle, R. A., J. R. Murphy, and J. Schaeffer (2003), Orbital change experiments with a Mars general circulation model, Icarus, 161(1), $66-89$.

Joshi, M. M., S. R. Lewis, P. L. Read, and D. C. Catling (1995), Western boundary currents in the Martian atmosphere: Numerical simulations and observational evidence, J. Geophys. Res., 100, 5485-5500.

Kahn, R. A., T. Z. Martin, R. W. Zurek, and S. W. Lee (1992), The Martian dust cycle, in Mars, edited by H. Kieffer et al., pp. 1017-1053, Univ. of Ariz. Press, Tucson.

Kahre, M. A., J. R. Murphy, R. M. Haberle, F. Montmessin, and J. Schaeffer (2005), Simulating the Martian dust cycle with a finite surface dust reservoir, Geophys. Res. Lett., 32, L20204, doi:10.1029/2005GL023495.

Leovy, C. B., J. B. Pollack, B. A. Smith, E. N. Shipley, R. L. Wildey, A. T. Young, and G. A. Briggs (1972), Mariner 9 television experiment progress report, Icarus, 17, 373.

Leovy, C. B., R. W. Zurek, and J. B. Pollack (1973), Mechanisms for Mars dust storms, J. Atmos. Sci., 30, 749-762.

Lindzen, R. S., and A. Y. Hou (1988), Hadley circulations for zonally averaged heating centered off the equator, J. Atmos. Sci., 45, 2416-2427.

Liu, J., M. I. Richardson, and R. J. Wilson (2003), An assessment of the global, seasonal, and interannual spacecraft record of Martian climate in the thermal infrared, J. Geophys. Res., 108(E8), 5089, doi:10.1029/ 2002JE001921.

Martin, L. J., and R. W. Zurek (1993), An analysis of the history of dust activity on Mars, J. Geophys. Res., 98, 3221-3246.

Martin, T. Z., and M. I. Richardson (1993), New dust opacity mapping from Viking infrared thermal mapper data, J. Geophys. Res., 98, 10,94110,949 .

Mischna, M. A., M. I. Richardson, R. J. Wilson, and D. J. McCleese (2003) On the orbital forcing of Martian water and $\mathrm{CO}_{2}$ cycles: A general circulation model study with simplified volatile schemes, J. Geophys. Res., 108(E6), 5062, doi:10.1029/2003JE002051.

Murphy, J. R., R. M. Haberle, O. B. Toon, and J. B. Pollack (1993), Martian global dust storms: Zonally symmetric numerical simulations including size dependent particle transport, J. Geophys. Res., 98, 3197-3220.

Murphy, J. R., O. B. Toon, R. M. Haberle, and J. B. Pollack (1995), Numerical simulations of the decay of Martian global dust storms, J. Geophys. Res., 104, 24,177-24,194.

Newman, C. E., S. R. Lewis, P. L. Read, and F. Forget (2002a), Modeling the Martian dust cycle: 1. Representations of dust transport processes, J. Geophys. Res., 107(E12), 5123, doi:10.1029/2002JE001910.

Newman, C. E., S. R. Lewis, P. L. Read, and F. Forget (2002b), Modeling the Martian dust cycle: 2. Multiannual radiatively active dust transport simulations, J. Geophys. Res., 107(E12), 5124, doi:10.1029/ 2002JE001920.

Newman, C. E., P. L. Read, and S. R. Lewis (2005), Investigating atmospheric predictability on Mars using breeding vectors in a general circulation model, Q. J. R. Meteorol. Soc., 130, 2971-2989, doi:10.1256 qj.03.209.

Pankine, A. A., and A. P. Ingersoll (2002), Interannual variability of Martian global dust storms: Simulations with a low order model of the general circulation, Icarus, 155, 299-323

Pankine, A. A., and A. P. Ingersoll (2004), Interannual variability of Mars global dust storms: An example of self organized criticality?, Icarus, 170(2), 514-518.
Renno, N. O., M. L. Burket, and M. P. Larkin (1998), A simple thermodynamic theory for dust devils, J. Atmos. Sci., 55, 3244-3252.

Renno, N. O., A. A. Nash, J. Lunine, and J. Murphy (2000), Martian and terrestrial dust devils: Test of a scaling theory using Pathfinder data, J. Geophys. Res., 105, 1859-1865.

Ruff, S. W., and P. R. Christensen (2002), Bright and dark regions on Mars: Particle size and mineralogical characteristics based on Thermal Emission Spectrometer data, J. Geophys. Res., 107(E12), 5127, doi:10.1029/ 2001JE001580

Silli, T., R. M. Haberle, and J. R. Murphy (1997), Sensitivity of Martian southern polar cap edge winds and surface stresses to dust optical depth thickness and the large scale sublimation flow, Adv. Space Res., 19 $1241-1244$

Smith, M. D. (2004), Interannual variability in TES Atmospheric observations of Mars during 1999-2003, Icarus, 108, 148-165.

Smith, M. D., B. J. Conrath, J. C. Pearl, and P. R. Christensen (2002), Thermal Emission Spectrometer observations of Martian planet-encircling dust storm 2001A, Icarus, 157, 259-263.

Strausberg, M. J., H. Wang, M. I. Richardson, S. P. Ewald, and A. D. Toigo (2005), Observations of the initiation and evolution of the 2001 Mars global dust storm, J. Geophys. Res., 110, E02006, doi:10.1029/ 2004JE002361

Wang, H., and A. P. Ingersoll (2002), Martian clouds observed by Mars Global Surveyor Mars Orbiter Camera, J. Geophys. Res., 107(E10), 5078, doi:10.1029/2001JE001815.

Wang, H., M. I. Richardson, R. J. Wilson, A. P. Ingersoll, A. D. Toigo, and R. W. Zurek (2003), Cyclones, tides, and the origin of a cross-equatorial dust storm on Mars, Geophys. Res. Lett., 30(9), 1488, doi:10.1029/ 2002GL016828.

Wang, H., R. W. Zurek, and M. I. Richardson (2005), Relationship between frontal dust storms and transient eddy activity in the northern hemisphere of Mars as observed by Mars Global Surveyor, J. Geophys. Res., 110, E07005, doi:10.1029/2005JE002423.

Wilson, R. J. (1997), A general circulation model simulation of the Martian polar warming, Geophys. Res. Lett., 24, 123-127.

Wilson, R. J., and K. Hamilton (1996), Comprehensive model simulations of thermal tides in the Martian atmosphere, J. Atmos. Sci., 53, $1290-$ 1326

Wilson, R. J., and M. I. Richardson (1999), Comparison of Mars GCM with Viking mission observations, in Fifth International Conference on Mars, Abstract 6234, Lunar and Planet. Inst., Houston, Tex.

Wilson, R. J., and M. I. Richardson (2000), The Martian atmosphere during the Viking mission, 1. Infrared measurements of atmospheric temperatures revisited, Icarus, 145, 555-579.

Wilson, R. J., D. Banfield, B. J. Conrath, and M. D. Smith (2002), Traveling waves in the Northern Hemisphere of Mars, Geophys. Res. Lett., 29(14), 1684, doi:10.1029/2002GL014866.

Zurek, R. W. (1981), Inference of the dust opacities for the 1977 Martian great dust storms from Viking Lander 1 pressure data, Icarus, 45, 202 215 .

Zurek, R. W., and C. B. Leovy (1981), Thermal tides in the dusty Martian atmosphere: A verification of theory, Science, 213, 437-439.

Zurek, R. W., and L. J. Martin (1993), Interannual variability of planetencircling dust storms on Mars, J. Geophys. Res., 98(E2), 3247-3259.

A. Ingersoll and M. Richardson, Division of Geological and Planetary Sciences, California Institute of Technology, MC 150-21 Caltech, 1200 East California Boulevard, Pasadena, CA 91125, USA.

S. Basu, Department of Atmospheric Science, Texas A\&M University, 3150 TAMU, College Station, TX 77843, USA. (shabari_basu@yahoo. com)

J. Wilson, Geophysical Fluid Dynamics Laboratory, National Oceanic and Atmospheric Administration, P.O. Box 308, Princeton, NJ 08542, USA. 